Research Paper

\title{
Characterizing long COVID in an international cohort: 7 months of symptoms and their impact
}

\author{
Hannah E. Davis ${ }^{\mathrm{a}, 1}$, Gina S. Assaf ${ }^{\mathrm{a}, 1}$, Lisa McCorkell ${ }^{\mathrm{a}, 1}$, Hannah Wei ${ }^{\mathrm{a}, 1}$, Ryan J. Low ${ }^{\mathrm{a}, \mathrm{b}, 1}$, \\ Yochai Re'em ${ }^{\mathrm{a}, \mathrm{c}, 1}$, Signe Redfield ${ }^{\mathrm{a}}$, Jared P. Austin ${ }^{\mathrm{a}, \mathrm{d}}$, Athena Akrami ${ }^{\mathrm{a}, \mathrm{b}, 1, *}$ \\ a Patient-Led Research Collaborative \\ ${ }^{\mathrm{b}}$ Sainsbury Wellcome Centre, University College London, London, United Kingdom \\ ${ }^{\mathrm{c}}$ Department of Psychiatry, NewYork-Presbyterian Hospital / Weill Cornell Medicine, NYC, United States \\ ${ }^{\mathrm{d}}$ Oregon Health and Science University, Portland, OR, United States
}

\section{A R T I C L E I N F O}

\section{Article History:}

Received 2 April 2021

Revised 18 June 2021

Accepted 21 June 2021

Available online $\mathrm{xxx}$

\section{Keywords:}

Long COVID

Post-acute Sequelae of COVID-19

PASC

Patient-Led research

COVID-19

COVID recovery

COVID-19 symptoms

Post-COVID-19 Syndrome

Post Acute COVID

Long Hauler

\begin{abstract}
A B S T R A C T
Background: A significant number of patients with COVID-19 experience prolonged symptoms, known as Long COVID. Few systematic studies have investigated this population, particularly in outpatient settings. Hence, relatively little is known about symptom makeup and severity, expected clinical course, impact on daily functioning, and return to baseline health.

Methods: We conducted an online survey of people with suspected and confirmed COVID-19, distributed via COVID-19 support groups (e.g. Body Politic, Long COVID Support Group, Long Haul COVID Fighters) and social media (e.g. Twitter, Facebook). Data were collected from September 6, 2020 to November 25, 2020. We analyzed responses from 3762 participants with confirmed (diagnostic/antibody positive; 1020) or suspected (diagnostic/antibody negative or untested; 2742) COVID-19, from 56 countries, with illness lasting over 28 days and onset prior to June 2020. We estimated the prevalence of 203 symptoms in 10 organ systems and traced 66 symptoms over seven months. We measured the impact on life, work, and return to baseline health.

Findings: For the majority of respondents (>91\%), the time to recovery exceeded 35 weeks. During their illness, participants experienced an average of 55.9+/- 25.5 (mean+/-STD) symptoms, across an average of 9.1 organ systems. The most frequent symptoms after month 6 were fatigue, post-exertional malaise, and cognitive dysfunction. Symptoms varied in their prevalence over time, and we identified three symptom clusters, each with a characteristic temporal profile. $85.9 \%$ of participants (95\% CI, 84.8\% to 87.0\%) experienced relapses, primarily triggered by exercise, physical or mental activity, and stress. $86.7 \%$ (85.6\% to $92.5 \%$ ) of unrecovered respondents were experiencing fatigue at the time of survey, compared to $44.7 \%$ (38.5\% to $50.5 \%$ ) of recovered respondents. 1700 respondents (45.2\%) required a reduced work schedule compared to pre-illness, and an additional 839 (22.3\%) were not working at the time of survey due to illness. Cognitive dysfunction or memory issues were common across all age groups ( $88 \%)$. Except for loss of smell and taste, the prevalence and trajectory of all symptoms were similar between groups with confirmed and suspected COVID-19.

Interpretation: Patients with Long COVID report prolonged, multisystem involvement and significant disability. By seven months, many patients have not yet recovered (mainly from systemic and neurological/cognitive symptoms), have not returned to previous levels of work, and continue to experience significant symptom burden.

Funding: All authors contributed to this work in a voluntary capacity. The cost of survey hosting (on Qualtrics) and publication fee was covered by AA's research grant (Wellcome Trust/Gatsby Charity via Sainsbury Wellcome center, UCL).
\end{abstract}

(C) 2021 The Authors. Published by Elsevier Ltd. This is an open access article under the CC BY license

(http://creativecommons.org/licenses/by/4.0/)

\footnotetext{
* Corresponding author.

E-mail address: athena.akrami@ucl.ac.uk (A. Akrami).

1 These authors contributed equally.
}

\section{Introduction}

Public discourse on COVID-19 has largely centered around those with severe or fatal illness [1]. However, recent studies show that a 


\section{Research in context}

\section{Evidence before this study}

Several studies have confirmed the presence of persistent symptoms following acute infection with COVID-19. Most recently, a large study conducted within the United States Veteran Affairs Health Care System found that patients with acute COVID-19 experienced higher rates of morbidity and mortality over the ensuing six months following diagnosis compared to uninfected individuals. This study, and many preceding it, utilized administrative databases and ICD-10 codes to identify and categorize these sequelae, which may inadvertently simplify the complexity of the Long COVID patient experience and miss details that can only be captured through direct patient assessment.

\section{Added value of this study}

This patient-directed study examines the largest collection of symptoms identified in the Long COVID population to date, is the first to quantify individual symptom trajectories over an extended period of time, and demonstrates the large impact symptoms have on patients' ability to work and perform daily tasks. The comprehensive assay of symptoms spans 10 organ systems (neuropsychiatric, systemic, reproductive, cardiovascular, musculoskeletal, immunological, head-ear-eye-nosethroat, pulmonary, gastrointestinal, and dermatologic). Cluster analysis reveals that symptoms share common modes of variation in their prevalence over time, and that symptoms with similar time courses are distributed across multiple organ systems. A combination of the neurological/cognitive and systemic symptoms are shown to persist the longest.

\section{Implications of all the available evidence}

Given the millions of cases of COVID-19 worldwide and current research showing one in seven COVID-19 patients still symptomatic at 12 weeks, the number of Long COVID patients is likely substantial. The results of this study suggest Long COVID is composed of heterogeneous sequelae that often affect multiple organ systems, with significant impacts on morbidity, mortality, and quality of life. Given the heterogeneity of Long COVID, multidisciplinary research will be necessary to understand the pathophysiology of the disease and develop effective treatments. This research also highlights the importance of slowing the spread of COVID-19 through validated public health measures and vaccinations, and highlights the necessity of a robust safety net including sick leave, family leave, disability benefits, and workplace protections and flexibilities.

growing number of patients with initially mild COVID-19 will experience prolonged symptoms [2,3], the profile and timeline of which remains uncertain [2,4-9]. Early in the course of the pandemic, patients identified this trend, referring to themselves as "Long-Haulers" and the prolonged illness as "Long COVID"[10]. There exist few systematic studies investigating this population, and relatively little is known about the range of symptom makeup and severity, expected clinical course, impact on daily functioning, and expected return to baseline health [11].

While as of yet there is no agreed upon case definition of Long COVID $[8,12]$, we define the illness as a collection of symptoms that develop during or following a confirmed or suspected case of COVID19 , and which continue for more than 28 days. This is a similar definition to the Centers for Disease Control and Prevention's (CDC) "Post-COVID conditions"[13].

In this paper, we report results from an online survey investigating the symptoms of Long COVID in patients with illness onset between December 2019 and May 2020, allowing analysis of symptoms over 7 months' duration. The aim of this study is to better describe the patient experience and recovery process in those with confirmed or suspected COVID-19 illness, with a specific emphasis on the Long COVID experience. The unique approach of this study utilizes patient-driven research [14] in order to establish a foundation of evidence for medical investigation, improvement of care $[15,16]$, and advocacy for the Long COVID population. In this study, we investigate the patient's lived experience, emphasizing symptom course and severity over time with an in-depth look into neurological and neuropsychiatric symptoms, recovery, and return to baseline, including work impact. Other topics investigated in the survey will be included in future reports.

\section{Methods}

\subsection{Study design}

The survey was created by a team of patients with COVID-19 who are members of the Body Politic online COVID-19 support group and formed the Patient-Led Research Collaborative. The group conducted its first survey in April 2020 and issued a subsequent report in May 2020 [7]. The second survey was created to investigate details of recovery, testing results, the impact on mental health, and a more comprehensive set of symptoms with a greater emphasis on neurological symptoms. During the curation of survey questions, we worked closely with other patients to compile the list of symptoms, design research questions on how the Long COVID condition may affect daily life of the patients, and optimize the questionnaire design to reduce survey fatigue.

The survey was launched on September 6, 2020. Data were collected using Qualtrics (www.qualtrics.com), an online survey platform. All respondents gave digital informed consent prior to participating. Survey responses contained no personally identifiable information, and email addresses collected for survey distribution were encrypted as anonymized participant IDs. The study was approved by the University College London (UCL) Research Ethics Committee [16,159.002] (London, UK), and Oregon Health and Science University Institutional Review Board (IRB) (Portland, OR, USA), with UCL serving as the primary site. The Weill Cornell Medical College IRB determined non-engagement.

The survey consisted of 257 questions and required a median time of $69.3 \mathrm{~min}$ to complete. To account for Long COVID symptoms that limit sustained focus and attention, respondents were encouraged to take breaks while completing the survey. Progress was saved for up to 30 days to allow respondents to return to the survey at a later time. Questions that mentioned technical terms included a description in plain language.

The survey was created in English and translated into eight additional languages: Spanish, French, Portuguese, Italian, Dutch, Russian, Bahasa Indonesian, and Arabic. Links to the survey were disseminated via email, social media, and the online patient support groups listed in Appendix C.4. Data included in the analysis were collected from September 6 to November 25, 2020.

\subsection{Study population (Inclusion criteria)}

The survey "Information Sheet" (accessible here: patientresearch covid19.com/survey2) stated: "You are being invited to participate in this research study because you have had a COVID-19, or suspected COVID-19 infection (still suffering or suffered symptoms) for longer than 1 week and you are 18 years of age or older." All respondents consented to these criteria. To characterize Long COVID symptoms 
Table 1

Testing status.

\begin{tabular}{|c|c|c|c|c|}
\hline Type of SARS-CoV-2 Test* & Number of Respondents Tested & $\%$ of Respondents Tested ${ }^{* *}$ & $\begin{array}{l}\text { Number of Respondents with } \\
\text { Positive Results }\end{array}$ & $\begin{array}{l}\% \text { of Respondents with Positive } \\
\text { Results** }\end{array}$ \\
\hline Diagnostic (RT-PCR/antigen) & $2330^{* * *}$ & $61.9 \%$ & 600 & $15.9 \%$ \\
\hline Antibody (IgG, IgM or both) & 2166 & $57.6 \%$ & 683 & $18.2 \%$ \\
\hline $\begin{array}{l}\text { Diagnostic (RT-PCR/antigen) or } \\
\text { Antibody }\end{array}$ & 3121 & $83.0 \%$ & 1020 & $27.1 \%$ \\
\hline
\end{tabular}

over an extended period, analysis was limited to respondents with illness lasting longer than 28 days and symptom onset between December 2019 and May 2020.

Methods used to distribute the survey did not allow us to determine the number of people who viewed the invitation. The proxy response rate was measured as the ratio of those who completed to those who started the survey. A total of 7285 responses were downloaded from the Qualtrics server on November 25, 2020. The following responses were removed from the dataset: incomplete (those not reaching the end of the survey, $n=2367$ ), no illness onset date $(n=2)$, onset date before December $2019(n=26), 0$ days of symptoms $(n=1)$, duplicate participants $(n=150)$, symptoms for 28 days or less $(n=401)$, and illness onset after May $2020(n=576)$. This resulted in complete data from 3762 respondents.

One of the questions in the survey asked about the annual income of the participant's household. Options were provided based on five income quintiles in USA (USD), Canada (CAD), United Kingdom (GBP), and Europe (EURO). 3084 (82.0\%) respondents reported their income at the time of the survey, from which their socioeconomic status was estimated (Appendix C.1, Figure S1).

In addition to positively tested subjects [ $n=1020$, either diagnostic (RT-PCR/antigen) or antibody, Table 1], we included participants with absent $(n=1819)$ or negative test results $(n=923$, diagnostic and antibody). Comparison between these groups, in terms of symptom prevalence, symptom trajectory, and disease duration is reported in the Results section.

\subsection{Outcomes}

In this study we quantified disease duration, as well as symptom prevalence, probability time-course, severity, count, onset time, and temporal clustering. We also measured fatigue using the Fatigue Assessment Scale [17,18]. Return to baseline and working status were also measured.

The 203 symptoms (Appendix A) investigated were sourced from a combination of prior research, existing case-reports, literature review, and content shared by patients within support groups and on social media.

\subsection{Statistics and data analysis}

All statistics and data analysis were performed in MATLAB 2017a and 2020a, using a combination of built-in library functions and custom code.

\subsubsection{Survival function}

To investigate disease duration, the survey asked respondents to indicate the number of days their symptoms lasted. For non-recovered respondents, this number provided only a lower bound on the eventual duration of symptoms. To account for this censoring in the data, we characterized the distribution of durations using the Kaplan-Meier estimator[19]. The resulting survival function (Fig. 1a,
Supplemental Figure S2a) measures the probability that symptoms will persist beyond any specified amount of time.

\subsubsection{Prevalence estimation}

203 symptoms (Appendix A) were investigated by identifying their presence or absence. For 74 of these symptoms, respondents indicated at which points in their illness (weeks 1-4, months 2-7) they experienced the symptom, if at all. For each of the other 131 symptoms, participants indicated whether they had experienced the symptom at any point throughout the duration of their illness. Prevalence estimates were calculated by dividing the number of those who reported experiencing a symptom-either at a given time point (Fig. 4) or over the entire illness (Fig. 2, 3)-by the total number of participants to which the symptom applied (usually the whole sample, but occasionally out of a smaller population, such as respondents with menstrual cycles).

\subsubsection{Symptom time course estimation}

The survey asked respondents to detail their experience of a subset of 74 symptoms over time. Eight symptoms were excluded from analysis, as their measurement required specialized equipment or tests that many participants may not have had access to (Appendix A, Figure S5). Respondents indicated whether each of these symptoms was present during a series of time intervals following the onset of their first symptoms (weeks $1-4$, months $2-7$ ). The time course of each symptom was defined as the probability of experiencing the symptom in each time interval, given that: 1) recovery had not occurred prior to the end of the interval, and 2) the symptom was applicable (menstruation-related symptoms are presented only for menstruating respondents). Plotted time courses in Fig. 4 are linearly interpolated between the centers of each time interval.

Fig. 5

\subsubsection{Symptom severity and count}

Overall symptom severity for each time interval (weeks 1-4, month 2-7) was measured using a Likert scale (no symptom, very mild, mild, moderate, severe, very severe).

\subsubsection{Symptom onset analysis}

Continuous, piecewise-constant distributions were fit to onset times for each symptom using maximum likelihood and accounting for interval censoring (onset times for each respondent could only be measured up to the enclosing time intervals, described above). For each symptom, the estimated probability density at time $t$ was given by the fraction of respondents who first experienced the symptom in the interval containing $t$ (among those who experienced it at any point), divided by the duration of the interval. Mean onset time was calculated as the expected value of the estimated distribution.

\subsubsection{Symptom time course clustering}

Symptom time courses were clustered using spherical k-means, a variant of k-means based on cosine distances [20]. Each time course 
a. Probability of having symptoms after $T$

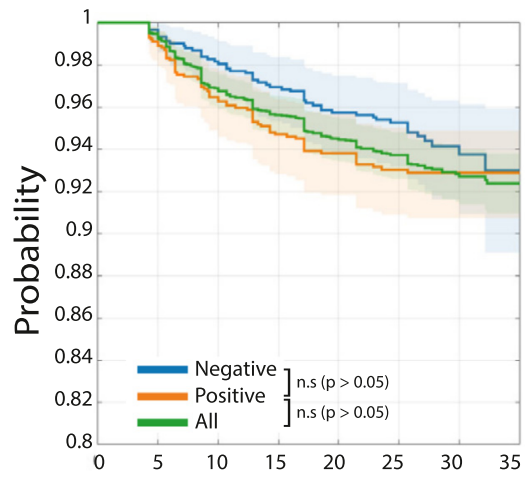

Weeks of illness (T) b. Symptom severity score over time

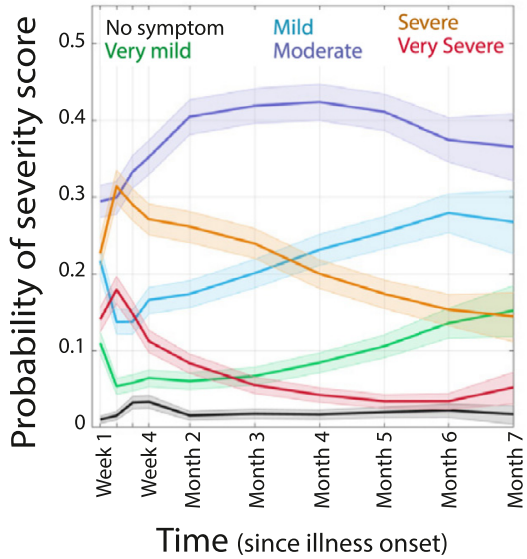

c. Average number of symptoms over time

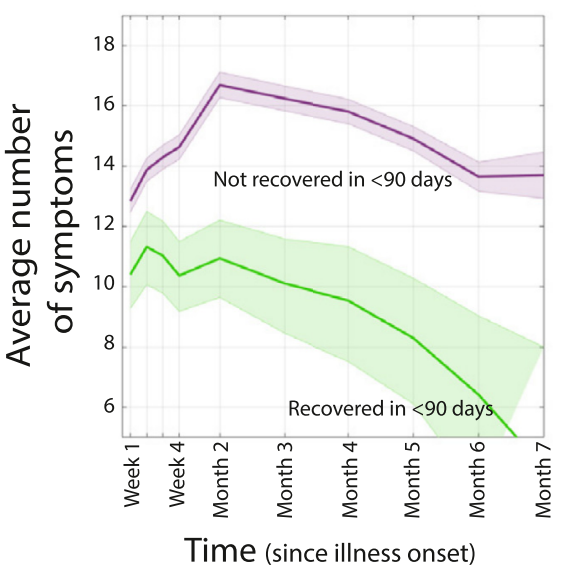

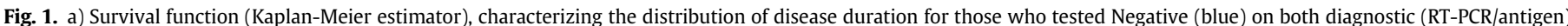

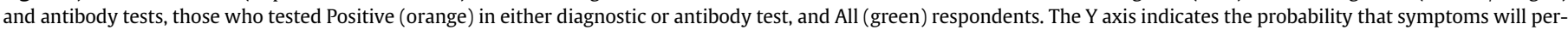

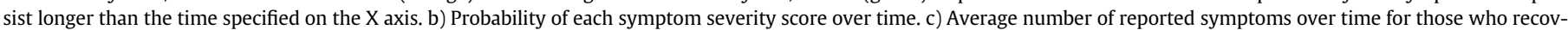

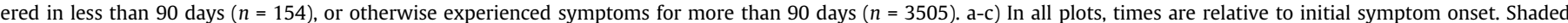
regions represent $95 \%$ simultaneous confidence bands.

is a 10-dimensional vector, representing the conditional probability of experiencing the symptom in each of the 10 time bins. The cosine distance is a monotonic function of the angle between vectors, and is insensitive to their magnitudes. Therefore, it is well suited to measuring differences between time course shapes (i.e. changes in relative amplitude over time), while remaining invariant to differences in overall symptom prevalence. We used a variant of Lloyd's algorithm designed for spherical k-means, with initialization by the k-means++ algorithm, and 100 random restarts to avoid suboptimal local minima. The number of clusters $(k=3)$ was chosen by hand, to provide a reasonable tradeoff between capturing structure in the data and obtaining a parsimonious explanation.

\subsubsection{Symptom time course sorting}

The heatmaps in Fig. 6 and Figure S3 show normalized symptom time courses, sorted such that similarly-shaped time courses appear nearby in the ordering. To compute the sort ordering, similarity between time courses was measured using the cosine distance, as above. Classical multidimensional scaling (MDS) was then used to embed time courses into a one-dimensional Euclidean space, such that pairwise distances in the embedding space approximated the given cosine distances. Time courses were sorted according to their order in the embedding space.

\subsubsection{Confidence intervals}

All confidence intervals and confidence bands were estimated using a nonparametric bootstrap approach with 10,000 iterations. Individual confidence intervals and pointwise confidence bands used the bias-corrected, accelerated (BCa) bootstrap[21]. Simultaneous confidence bands used the percentile bootstrap, with the percentile adjusted to give the correct simultaneous coverage probabilities.

\subsubsection{Fatigue assessment scale scores}

Fatigue Assessment Scale (FAS) scores were calculated based upon participants' subjective report during the "past one week." The scores were summarized into three categories $[17,18]$ : no fatigue (scores of $10-21)$, fatigue [22-34], and extreme fatigue ( $\geq 35)$.

See Appendix B for details of prevalence estimates, data stratification based on the diagnostic test time, and text analyses.

\subsection{Role of funding}

This study received no specific grant from any funding agency in the public, commercial, or not-for-profit sectors.

\section{Results}

\subsection{Demographics}

Survey response rate, calculated as the ratio of respondents who completed the survey [4] (918) to those who started the survey [7] (285), was $67.5 \%$. This study included 3762 survey respondents based on the eligibility criteria described in Methods. Detailed demographics are listed in Table 2. The majority of respondents were women $(78.9 \%$, significantly more than other genders, $p<0.001$, chisquared test), white ( $85.3 \%, p<0.001$, chi-squared test), and between the ages of 30 and 60 (33.7\% between ages $40-49,27.1 \%$ ages $50-59$, $26.1 \%$ ages $30-39$ ). A total of 56 countries were represented in the sample. Most of the respondents resided in the United States $(41.2 \%$, $p<0.01$, Tukey's HSD (honestly significant difference) multiple comparisons test). $91.9 \%$ of respondents completed the survey in English.

More than half of respondents $(56.7 \%, p<0.001$, chi-squared test) did not seek hospital-based care. $34.9 \%$ visited an ER or urgent care clinic but were not admitted to a hospital. $8.43 \%$ of respondents were hospitalized. $17.8 \%$ of respondents were healthcare workers (see Supplemental Material, Appendix C.2, for pre-existing conditions).

\subsection{Symptoms and severity over time}

\subsubsection{Symptom duration}

Respondents were considered recovered if they identified themselves as no longer experiencing symptoms at the time of survey completion. 257 respondents (6.8\%) recovered after day 28 of illness, and 3505 (93.2\%) were still experiencing symptoms at the time of survey completion.

A survival function, measuring the probability that symptoms will persist beyond any specified amount of time (see Methods), is shown in Fig. 1a. In this Long COVID cohort, the probability of symptoms lasting beyond 35 weeks was $91.8 \%$ (95\% confidence interval $89.5 \%$ to 93.5\%), with no statistically significant difference between positively (diagnostic/antibody) and negatively tested groups ( $p=0.18$, chisquared test), or men and women ( $p=0.49$, chi-squared test, Supplemental Figure S2a). Of the 3762 respondents, 2454 experienced 
a.
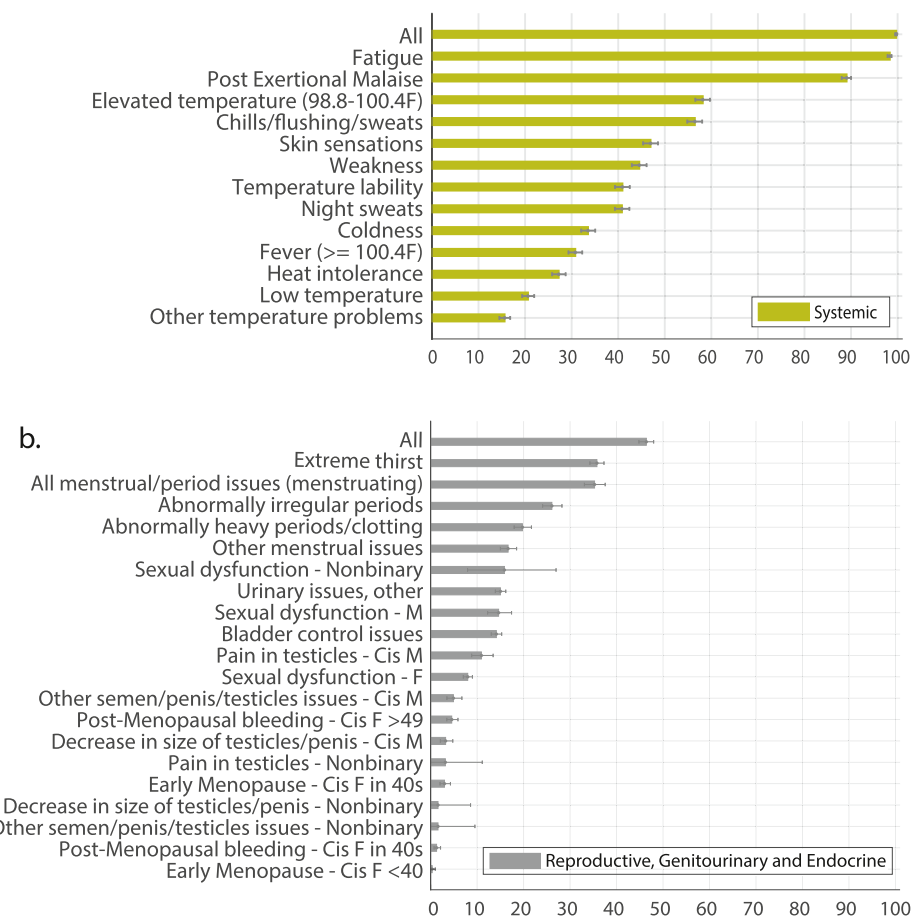

c.

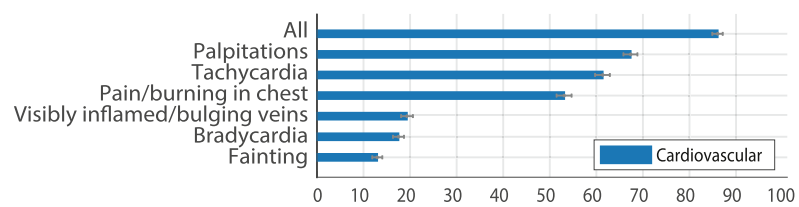

d.

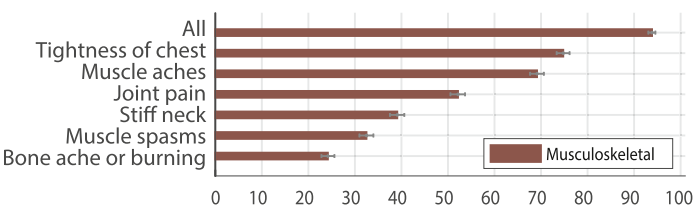

e.

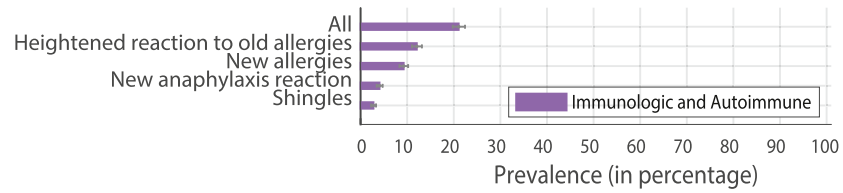

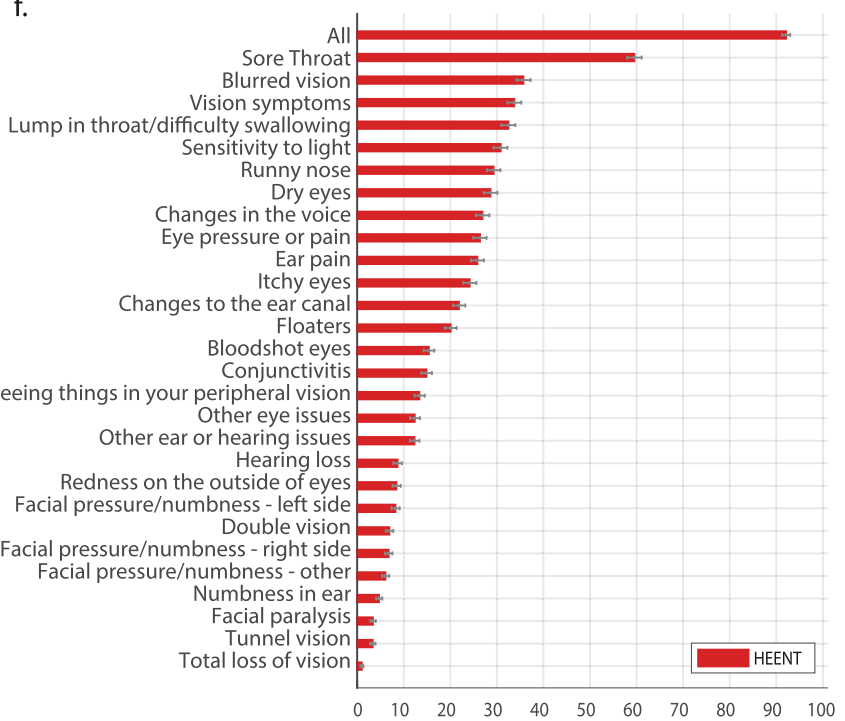

g.

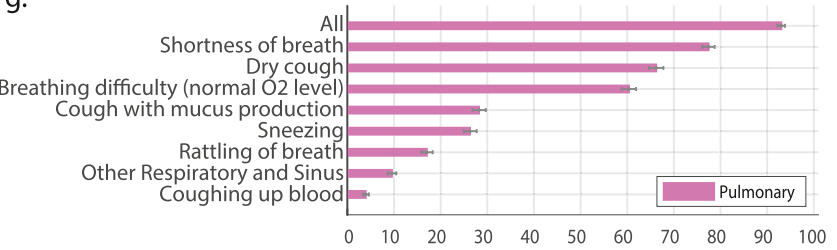

h.

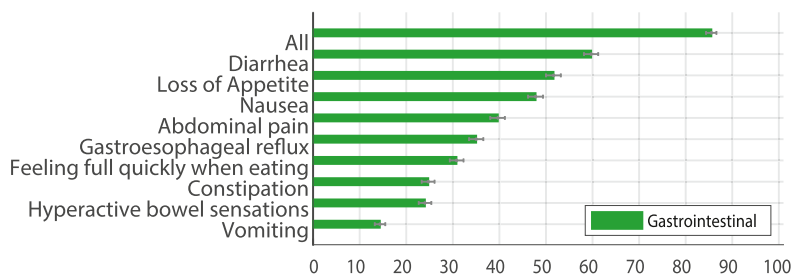

i.

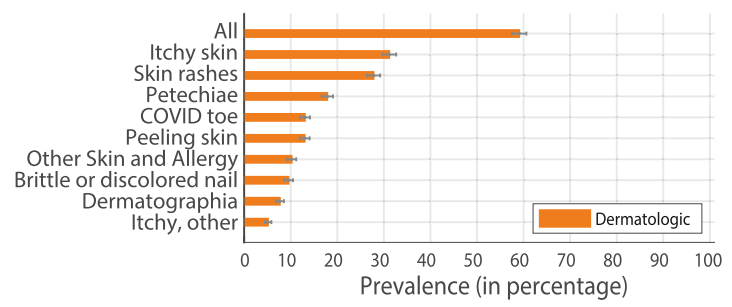

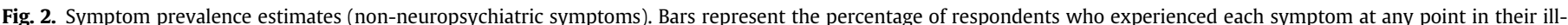

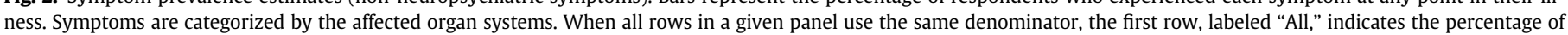

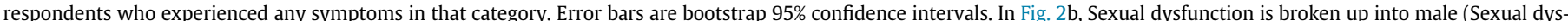

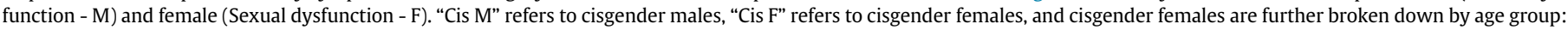
"Cis $F<40$ " indicates cisgender females age 39 or younger, "Cis $\mathrm{F}$ in 40 s" indicates cisgender females age 40 to 49 , and "Cis $F>49$ " indicates cisgender females age 50 or older.

symptoms for at least 180 days (six months). Among the remaining 1308 respondents, 233 recovered and the rest $(n=1075)$ took the survey before reaching six months of illness.

We described the Long COVID trajectory by assessing symptom severity and average number of symptoms over time. The probability of "severe" and "very severe" symptoms peaked during acute infection ( $<28$ days), while the probability of "moderate" and "mild" rose gradually thereafter (Fig. 1b).

In those who recovered in less than 90 days, the average number of symptoms peaked at week 2 [mean number of symptoms (out of 66 ): $11.35,95 \%$ confidence interval 13.58 to 9.44 ], and in those who did not recover in 90 days, the average number of symptoms peaked at month 2 (mean number of symptoms: $17.16,17.78$ to 16.54 ), with less decline over time (Fig. 1c, see Supplemental Figure S2 b-c for more comparisons between recovered and unrecovered participants). Respondents with symptoms for over six months experienced an average of 13.79 symptoms (95\% confidence interval 12.68 to 14.88 ) in month 7.

\subsubsection{Symptoms experienced at any point}

Overall symptom prevalence in 10 organ systems was estimated for a total of 203 symptoms (see Methods, Appendix A for list of 
a.

b.
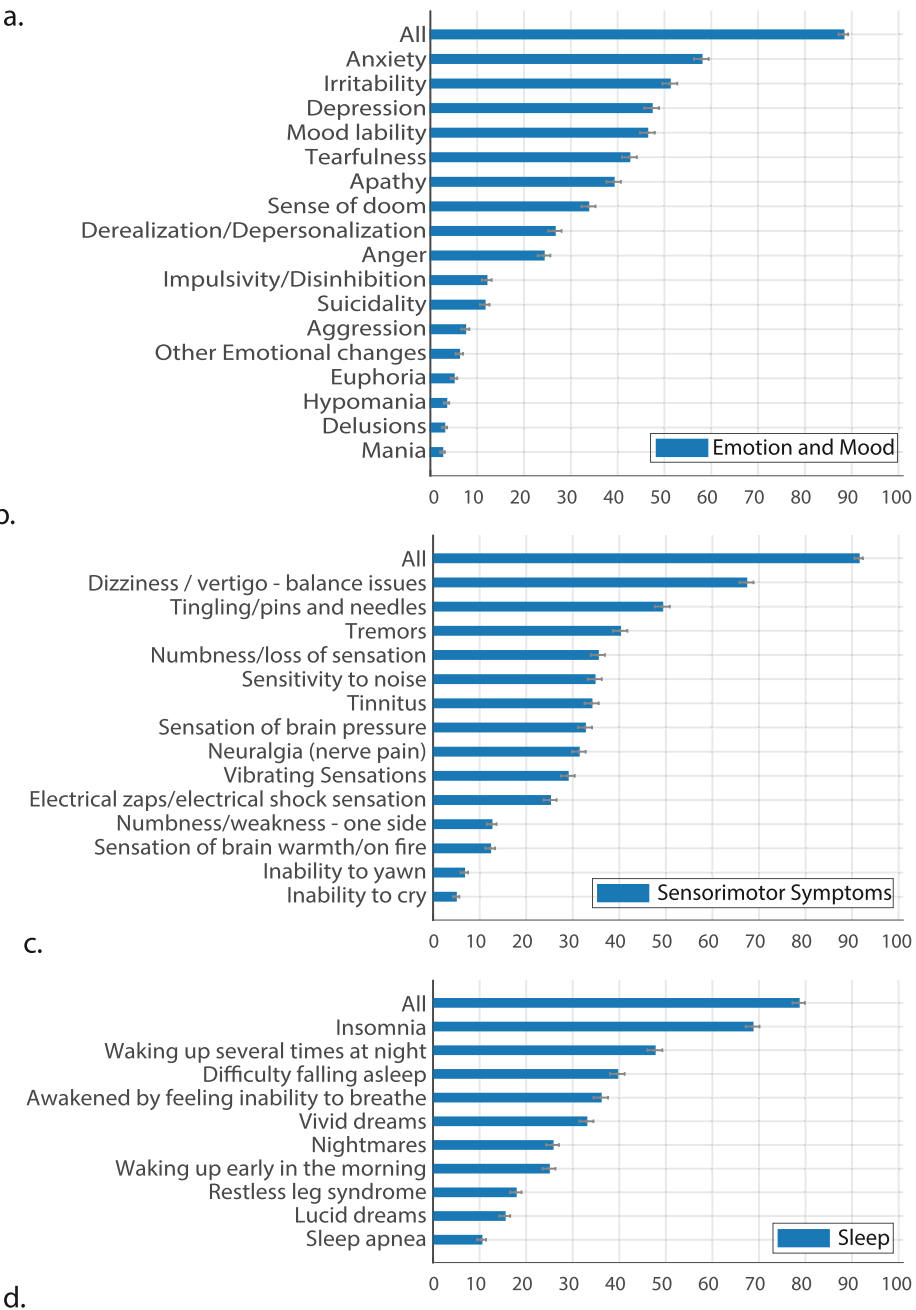

d.

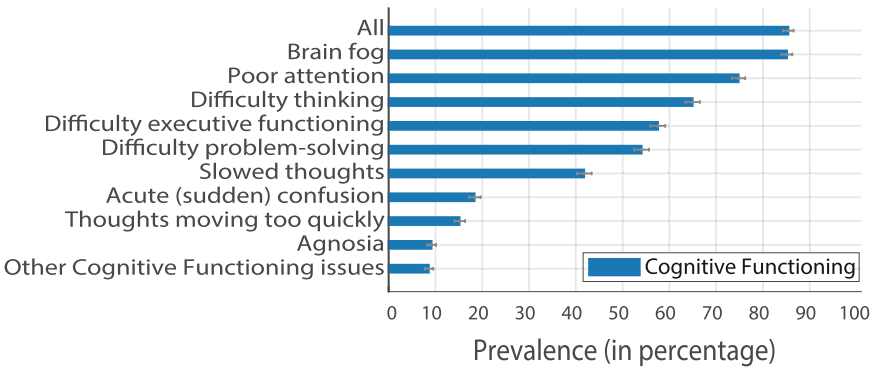

e.

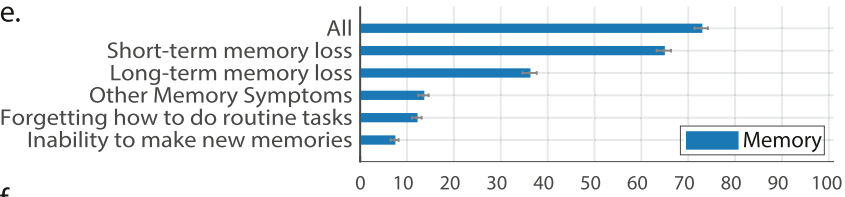

f.

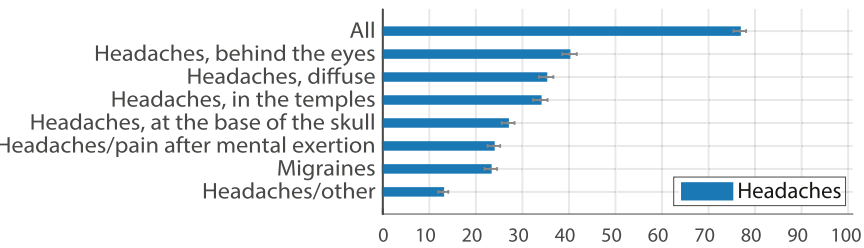

g.

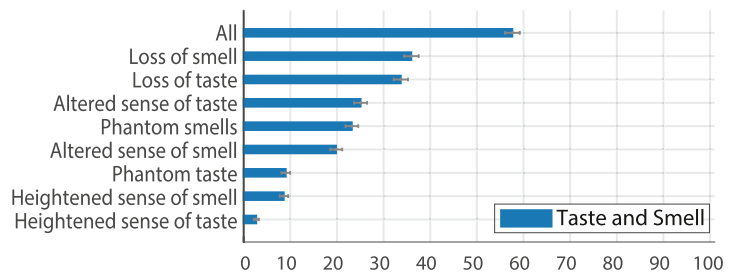

h.
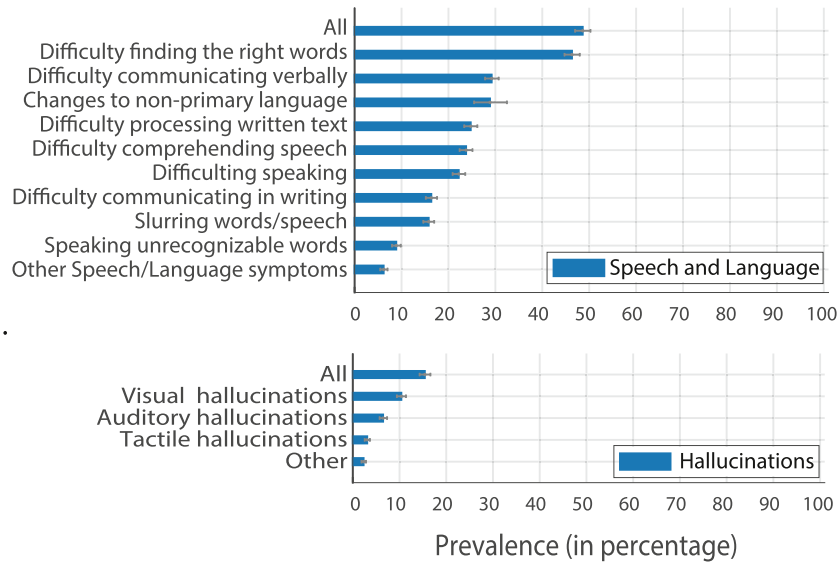

Prevalence (in percentage)

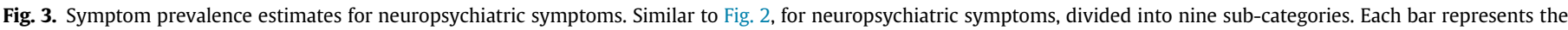
percentage of respondents who experienced that symptom. Error bars are bootstrap 95\% confidence intervals.

symptoms). Table 3 summarizes these prevalence estimates for 18 categories (nine non-neuropsychiatric organ systems: systemic, reproductive/genitourinary/endocrine, cardiovascular, musculoskeletal, immunological and autoimmune, HEENT, pulmonary, gastrointestinal and dermatologic in Fig. 2, and nine neuropsychiatric subgroups: cognitive dysfunction, speech and language, memory, headaches, smell and taste, sleep, emotion and mood, hallucinations, sensorimotor in Fig. 3, see Appendix F Table S6-S23 for raw data). Almost all participants experienced systemic (99.7\%, 95\% confidence interval $99.49 \%$ to $99.84 \%$ ), and HEENT (100\%) symptoms. Musculoskeletal, cardiovascular, gastrointestinal, pulmonary, and neuropsychiatric symptoms were prevalent in $>85 \%$ of participants (further detail in Supplemental Tables S5-S21). The top three most debilitating symptoms listed by patients were: 1$)$ fatigue $(n>2652), 2)$ breathing issues $(n>2242)$, and 3$)$ cognitive dysfunction $(n>1274)$.
Participants experienced an average of 55.9+/- 25.5 (mean+/-STD) symptoms during their illness.

\subsubsection{Symptoms over time}

Symptoms exhibited varying time courses, defined as the probability of experiencing each symptom at each time point, given that recovery had not yet occurred (Fig. 4). Most symptoms had a prolonged probability of occurrence throughout the seven month period (see Appendix F Table S24 for the raw data; Supplemental Figure S9 for male vs. female comparison).

Symptoms were clustered in three groups (Fig. 6), according to the shapes of their time courses (i.e. changes in relative amplitude over time, ignoring their overall prevalence, see Methods). Cluster 1 consists of symptoms that are most likely to occur early in the illness, reaching a high point in the first two or three weeks, then decreasing in probability over time. Cluster 2 consists of symptoms with a 


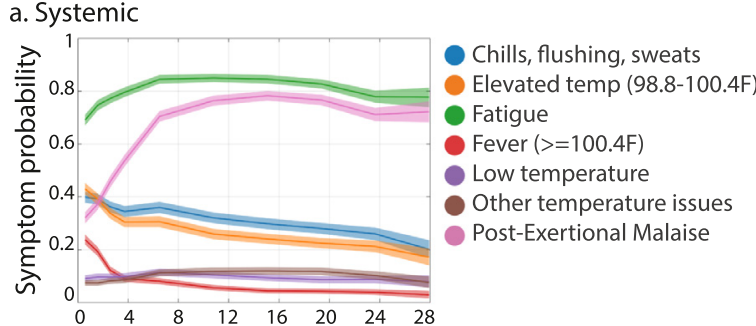

b. Reproductive, Genitourinary and Endocrine

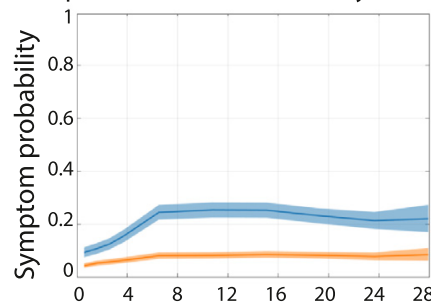

All menstrual, period issues

Bladder control issues

c. Cardiovascular

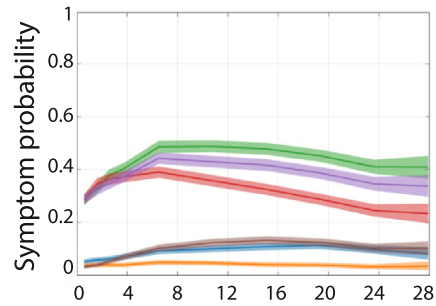

d. Musculoskletal

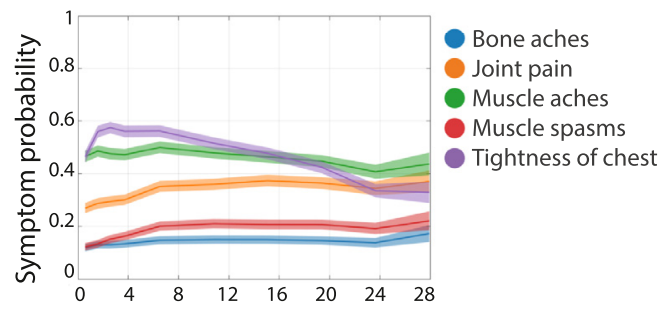

e. Immunologic and Autoimmune

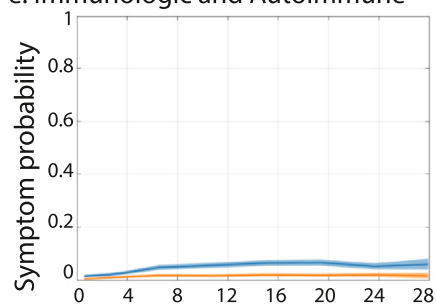

New allergies

New anaphylaxis reactions

f. Head, Ear, Eye, Nose, Throat (HEENT)

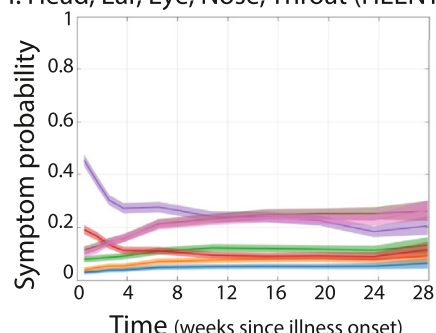

g. Pulmonary and Respiratory

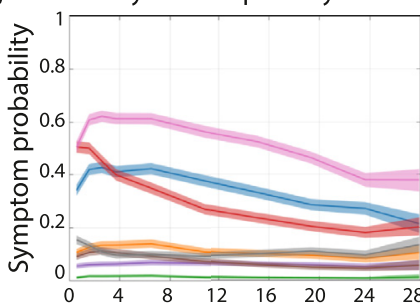

Breathing difficulties (normal O2)

Cough w mucus

Coughing up blood

Dry cough

Other respiratory, sinus issues

Rattling of breath

Shortness of breath

Sneezing

h. Gastrointestinal

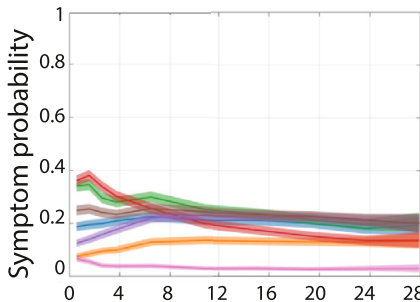

Abdominal pain

Constipation

- Diarrhea

Loss of appetite

Gastroesophageal reflux

Nausea

Vomiting

i. Dermatologic

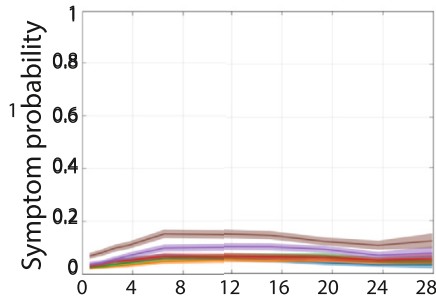

COVID toe

Dermatographia

Other allergy, skin issues

Peeling skin

Petechiae

Skin rashes

j. Neuropsychiatric - cognitive

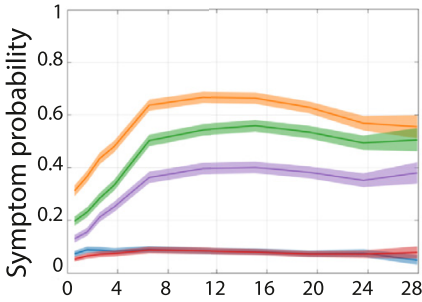

Acute confusion

Brain fog

Memory issues

- Slurring words

Speech and language issues

k. Neuropsychiatric - sensorimotor

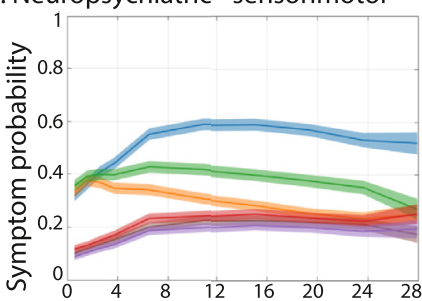

- Select sensorimotor symptom

Change of smell and taste

Dizziness and balance issues

- Neuralgia

- Tremors

Vibrating sensations
I. Neuropsychiatric - other

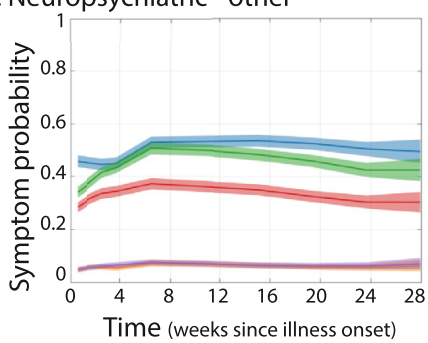

Hallucinations

- Headaches

Insomnia

Other sleeping issues

Sleep apnea

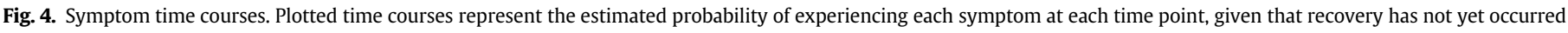

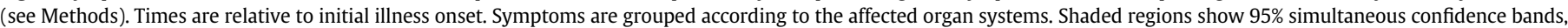
estimated separately for each symptom. 


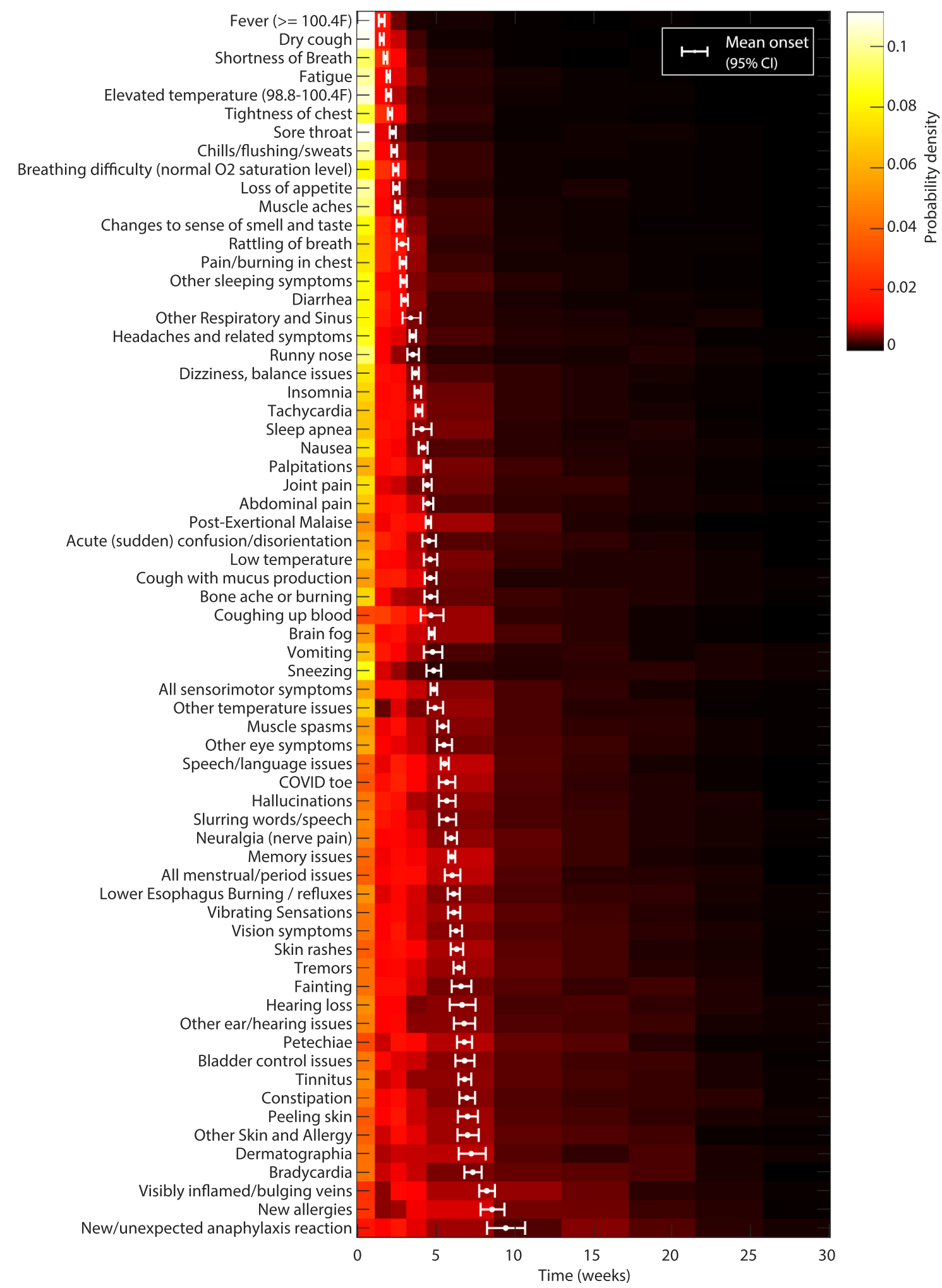

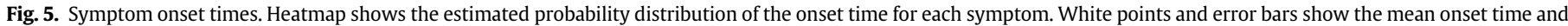
$95 \%$ pointwise confidence intervals. Symptoms are sorted by mean onset time.

relatively stable probability over time. Cluster 3 consists of symptoms most likely to increase sharply in the first two months. Their probability may plateau (like constipation), decrease slightly (like postexertional malaise and fatigue), or increase slightly in the later months (like tinnitus, hearing loss, muscle spasms, and tremors). All clusters contained symptoms from multiple organ systems, and Cluster 3 contained symptoms from all but one organ system (pulmonary/respiratory symptoms). A general progression from early to late symptoms can also be seen in the heatmap of normalized time courses (Fig. 6 \& Supplemental Figure S3), which have been sorted by similarity in shape (see Methods).

Symptom prevalence plots, together with the onset times and clusters (Figs. 2-6), show that symptoms spanned multiple organ systems. The mean number of organ systems affected in each 
Cluster 1

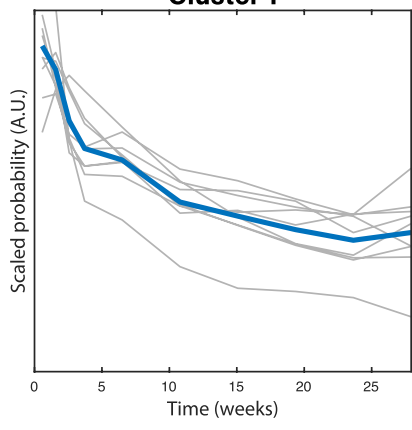

\begin{tabular}{ll}
\hline Cardiovascular \\
\hline Dermatologic \\
\\
\\
\hline Gastrointestinal & $\begin{array}{l}\text { 9. Diarrhea } \\
\text { 2. Loss of Appet } \\
\text { 4. Vomiting }\end{array}$ \\
\hline $\begin{array}{l}\text { HEENT } \\
\text { (Head, ears, }\end{array}$ & $\begin{array}{l}\text { 7. Runny nose } \\
\text { 6yes, nose Throat } \\
\text { throat) }\end{array}$ \\
\hline
\end{tabular}

Immunologic/

Musculoskeleta

Musculoskeletal

Neuropsychiatric
Cluster 2

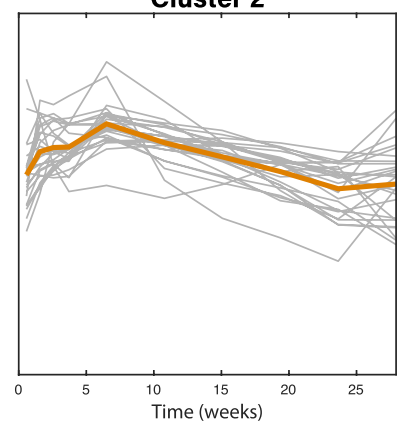

25. Fainting

19. Pain/burning in chest

33. Tachycardia

30. COVID toe

Cluster 3

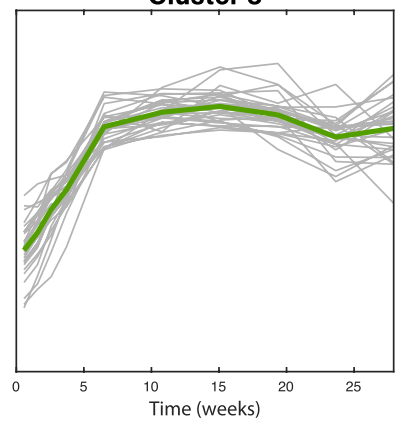

49. Bradycardia

38. Palpitations

64. Visibly inflamed/bulging veins

53. Dermatographia

55. Other Skin and Allergy

42. Peeling skin

54. Petechiae

44. Skin rashes

45. Constipation

43. Gastroesophageal reflux

18. Nausea

48. Hearing loss

51. Other ear/hearing issues

39. Other eye symptoms

58. Tinnitus

59. Vision symptoms

65. New allergies

63. New anaphylaxis reaction

32. Bone ache or burning 37. Joint pain

21. Muscle aches

40. Muscle spasms

15. Tightness of Chest

20. Acute (sudden) confusion/disorientation

12. Changes to sense of smell and taste

41. All sensorimotor symptoms

22. Dizzines, unsteadiness or balance issues

47. Brain fog

61. Memory issues

31. Hallucinations

29. Headaches and related symptoms

35. Insomnia

27. Other sleeping symptoms

50. Neuralgia (nerve pain)

62. Speech/language issues

52. Tremors

56. Vibrating Sensations

34. Sleep apnea

36. Slurring words/speech

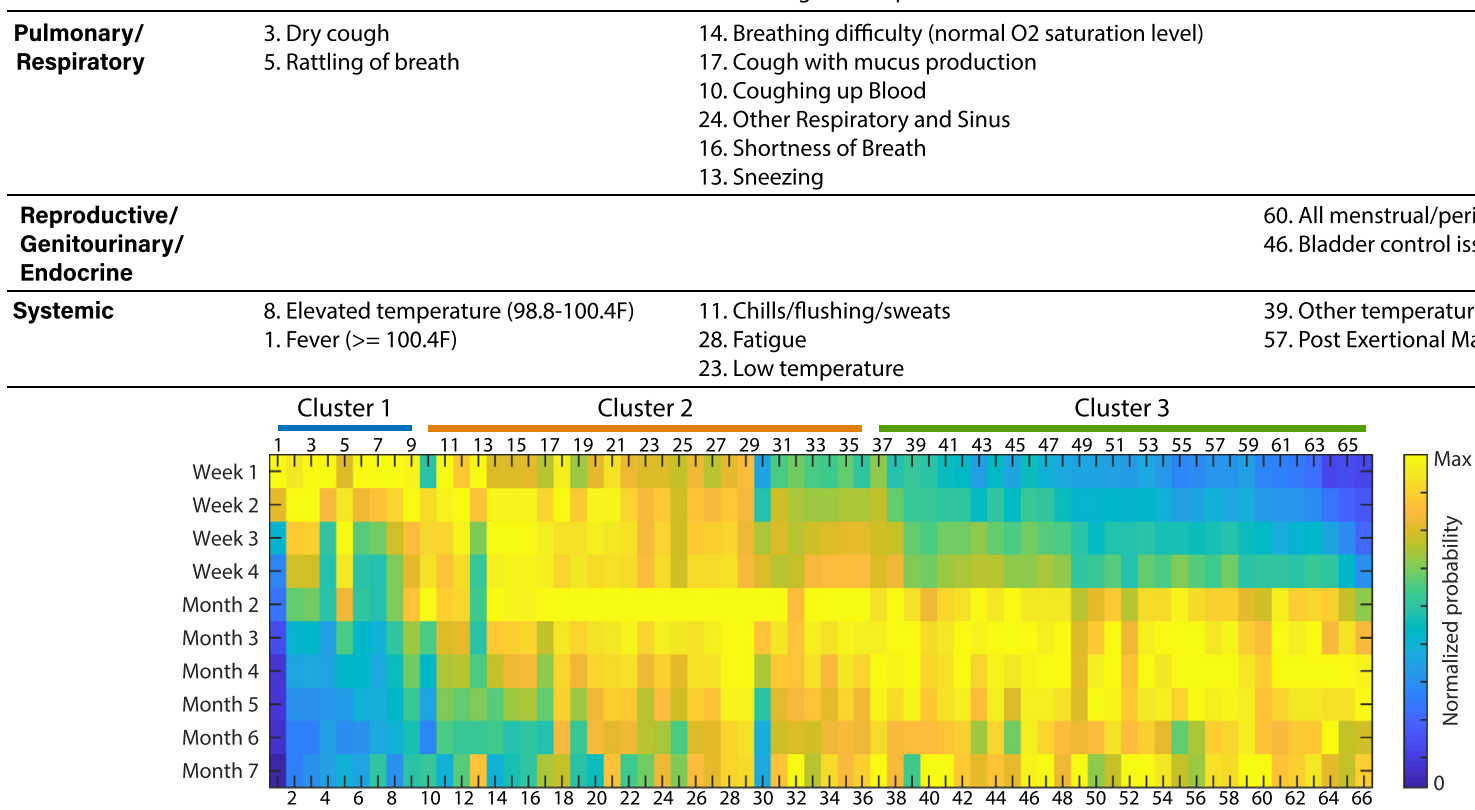

Fig. 6. Symptom clusters, based on temporal similarities. Plots (top row) show time courses for the symptoms in each cluster (in gray) and their mean (Cluster 1 in blue, Cluster 2 in orange, Cluster 3 in green). Time courses have been scaled separately for each symptom (by root mean squared amplitude) to visually compare their shapes. The table lists symptoms in each cluster, grouped by the affected organ systems. The heatmap (bottom row) shows time courses for all symptoms, sorted such that similarly shaped time courses are adjacent (see Methods). Columns have been scaled by their maximum amplitudes for visual comparison. Symptoms are numbered according to their table entries. 
Table 2

Demographics of survey respondents.

\begin{tabular}{|c|c|c|}
\hline Factor & $\begin{array}{l}\text { Number of Respondents } \\
(N=3762)\end{array}$ & $\%$ of Respondents \\
\hline \multicolumn{3}{|l|}{ Gender } \\
\hline Women* & 2969 & $78.9 \%$ \\
\hline Men* & 718 & $19.1 \%$ \\
\hline Nonbinary & 63 & $1.7 \%$ \\
\hline Other & 6 & $0.2 \%$ \\
\hline Preferred not to answer & 6 & $0.2 \%$ \\
\hline \multicolumn{3}{|l|}{ Age Group (years) } \\
\hline $18-29$ & 277 & $7.4 \%$ \\
\hline $30-39$ & 905 & $24.1 \%$ \\
\hline $40-49$ & 1166 & $31.0 \%$ \\
\hline $50-59$ & 937 & $25.0 \%$ \\
\hline $60-69$ & 380 & $10.1 \%$ \\
\hline $70-79$ & 85 & $2.3 \%$ \\
\hline $80+$ & 12 & $0.3 \%$ \\
\hline \multicolumn{3}{|l|}{ Ancestry** } \\
\hline White & 3418 & $85.3 \%$ \\
\hline $\begin{array}{l}\text { Hispanic, Latino, Spanish } \\
\text { Origin }\end{array}$ & 150 & $3.7 \%$ \\
\hline Asian, South Asian, SE Asian & 134 & $3.3 \%$ \\
\hline Black & 80 & $2.0 \%$ \\
\hline $\begin{array}{l}\text { Middle Eastern, North } \\
\text { African }\end{array}$ & 66 & $1.7 \%$ \\
\hline Indigenous Peoples & 50 & $1.6 \%$ \\
\hline Pacific Islander & 3 & $0.1 \%$ \\
\hline Other & 98 & $2.5 \%$ \\
\hline Prefer not to answer & 9 & $0.2 \%$ \\
\hline \multicolumn{3}{|l|}{ Environment } \\
\hline Urban & 1543 & $41.0 \%$ \\
\hline Suburban & 1586 & $42.2 \%$ \\
\hline Rural & 633 & $16.8 \%$ \\
\hline \multicolumn{3}{|l|}{ Country of Residence } \\
\hline USA & 1567 & $41.2 \%$ \\
\hline UK & 1316 & $35.0 \%$ \\
\hline France & 163 & $4.3 \%$ \\
\hline Canada & 155 & $4.1 \%$ \\
\hline Spain & 99 & $2.6 \%$ \\
\hline Netherlands & 61 & $1.6 \%$ \\
\hline Ireland & 58 & $1.5 \%$ \\
\hline Sweden & 55 & $1.5 \%$ \\
\hline Other & 288 & $7.7 \%$ \\
\hline \multicolumn{3}{|l|}{ Healthcare Worker } \\
\hline Yes & 668 & $17.8 \%$ \\
\hline No & 3094 & $82.2 \%$ \\
\hline \multicolumn{3}{|l|}{ Hospitalization } \\
\hline $\begin{array}{l}\text { Non-Hospitalized and no } \\
\text { visit to ER/Urgent Care }\end{array}$ & 2133 & $56.7 \%$ \\
\hline Visited ER or Urgent Care & 1312 & $34.9 \%$ \\
\hline Hospitalized & 317 & $8.4 \%$ \\
\hline
\end{tabular}

*Respondents included 2961 (78.7\%) cisgender women and $8(0.2 \%)$ transgender women, 714 (19.0\%) cisgender men and $4(0.1 \%)$ transgender men.

** Respondents were invited to select multiple ancestries. Percentages in this section are thus based on the total number of ancestries reported. $182(4.8 \%)$ respondents reported two ancestries, while $30(0.8 \%)$ reported three or more ancestries.

respondent was 9.08 (95\% confidence interval 9.04 to 9.13; see Symptom Details). Symptoms in the same organ-based category did not necessarily cluster together, and could appear across clusters. This indicates that symptoms affecting the same organ system can have differently shaped time courses and, conversely, symptoms affecting different organ systems can have similarly shaped time courses. Systemic and neurological/cognitive symptoms were the most likely to persist from disease onset to month 7 (see Symptom Details).

\subsection{Neuropsychiatric symptoms}

\subsubsection{Brain fog/Cognitive dysfunction and memory impairment}

$85.1 \%$ (95\% confidence interval $83.9 \%$ to $86.2 \%$ ) of respondents (3203) reported experiencing brain fog and cognitive dysfunction, including poor attention, executive functioning, problem solving, and
Table 3

Overall system prevalence data.

\begin{tabular}{|c|c|c|c|c|}
\hline Symptom Category & Total \# & $\begin{array}{l}\text { Mean } \\
\text { Prevalence }\end{array}$ & $\begin{array}{l}\text { Lower } \\
\mathrm{CI}\end{array}$ & Upper CI \\
\hline Systemic & 3750 & 99.70 & 99.49 & 99.84 \\
\hline $\begin{array}{l}\text { Reproductive / } \\
\text { Genitourinary / } \\
\text { Endocrine }\end{array}$ & 2341 & 62.25 & 60.68 & 63.74 \\
\hline Cardiovascular & 3236 & 86.04 & 84.90 & 87.16 \\
\hline Musculoskeletal & 3530 & 93.85 & 93.03 & 94.60 \\
\hline $\begin{array}{l}\text { Immunologic / } \\
\text { Autoimmune }\end{array}$ & 791 & 21.05 & 19.77 & 22.43 \\
\hline HEENT & 3761 & 100.00 & 100.00 & 100.00 \\
\hline $\begin{array}{l}\text { Pulmonary / } \\
\text { Respiratory }\end{array}$ & 3499 & 93.03 & 92.21 & 93.8 \\
\hline Gastrointestinal & 3216 & 85.50 & 84.37 & 86.6 \\
\hline Dermatologic & 2221 & 59.06 & 57.52 & 60.63 \\
\hline $\begin{array}{l}\text { Neuropsychiatric - } \\
\text { Cognitive } \\
\text { Dysfunction }\end{array}$ & 3212 & 85.43 & 84.29 & 86.55 \\
\hline $\begin{array}{l}\text { Neuropsychiatric - } \\
\text { Speech and } \\
\text { Language }\end{array}$ & 1828 & 48.62 & 47.00 & 50.21 \\
\hline $\begin{array}{l}\text { Neuropsychiatric - } \\
\text { Memory }\end{array}$ & 2739 & 72.81 & 71.40 & 74.20 \\
\hline $\begin{array}{l}\text { Neuropsychiatric - } \\
\text { Headaches }\end{array}$ & 2887 & 76.74 & 75.36 & 78.04 \\
\hline $\begin{array}{l}\text { Neuropsychiatric - } \\
\text { Smell and Taste }\end{array}$ & 2166 & 57.60 & 56.06 & 59.21 \\
\hline $\begin{array}{l}\text { Neuropsychiatric - } \\
\text { Sleep }\end{array}$ & 2955 & 78.58 & 77.25 & 79.88 \\
\hline $\begin{array}{l}\text { Neuropsychiatric - } \\
\text { Emotion and } \\
\text { Mood }\end{array}$ & 3320 & 88.25 & 87.19 & 89.26 \\
\hline $\begin{array}{l}\text { Neuropsychiatric - } \\
\text { Hallucinations }\end{array}$ & 580 & 15.42 & 14.30 & 16.64 \\
\hline $\begin{array}{l}\text { Neuropsychiatric - } \\
\text { Sensorimotor }\end{array}$ & 3440 & 91.44 & 90.48 & 92.29 \\
\hline
\end{tabular}

decision making (Fig. 3d, Supplemental Table S15 for prevalence of sub-symptoms). $72.8 \%$ ( $71.4 \%$ to $74.2 \%$ ) of all respondents (2739) experienced memory impairments, including both short-term and long-term memory loss (Fig. 3e \& Supplemental Table S16 for prevalence of sub-symptoms).

For $31.2 \%$ (29.7\% to $32.7 \%$ ) of respondents, the onset of brain fog/ cognitive dysfunction occurred in the first week of symptoms. Reports of cognitive dysfunction increased over the first three months, peaking at $66.7 \%$ (65.1\% to $68.2 \%)$, then decreased slightly in the following months. 55.5\% (52.5\% to 58.8\%) of month 7 respondents experienced cognitive dysfunction during month 7 (Fig. 4j).

The probability of experiencing memory symptoms increased the first few months, with $55.9 \%$ (54.3\% to $57.5 \%$ ) reporting memory symptoms in month $4.50 .5 \%$ (47.3\% to 53.6\%) of respondents with symptoms for over six months experienced memory symptoms in month 7 (also Fig. 4j).

Of those who experienced memory and/or cognitive dysfunction symptoms and had a brain MRI, $87 \%$ of the brain MRIs ( $n=345$, of 397 who were tested) showed no abnormalities.

3.3.1.1. Impact of cognitive dysfunction/memory on daily abilities and impact by age. $88.0 \%$ of the total respondents (3310) experienced cognitive dysfunction, memory loss, or both at similar rates across all age groups (Fig. 7a-c). The greatest area of impact reported was on work, with $86.2 \%$ (95\% confidence interval 84.4 to $88.0 \%$ ) of working respondents feeling mildly to severely unable to work (see Impact on Work section below for a discussion of the working status of respondents). See Fig. $7 d$ for the detailed list of memory and cognitive dysfunction impacts on daily life.

Selected quotes from respondents who described specific instances of memory loss or brain fog can be found in Appendix D. 
Table 5

Prevalence of sleep issues before and during illness.

\begin{tabular}{lll}
\hline Sleep Symptom & $\begin{array}{l}\text { Experienced During } \\
\text { Illness (of all participants) }\end{array}$ & $\begin{array}{l}\text { Had Symptom Before } \\
\text { Illness (of those who } \\
\text { experienced the symptom) }\end{array}$ \\
\hline Insomnia & $60 \%(67.1$ to $70.1 \%)$ & $21 \%$ \\
Night Sweats & $41 \%(39.2$ to $42.4 \%)$ & $16 \%$ \\
Awakened Feeling & $36 \%(34.5$ to $37.6 \%)$ & $\mathrm{N} / \mathrm{A}$ \\
$\quad$ Unable to Breathe & & \\
Restless Legs & $18 \%(16.6$ to $19 \%)$ & $14 \%$ \\
Sleep Apnea & $10 \%(9.5$ to $12.8 \%)$ & $34 \%$ \\
Vivid Dreams & $33 \%(31.5$ to $34.5 \%)$ & $23 \%$ \\
Nightmares & $26 \%(24.3$ to $27.1 \%)$ & $20 \%$ \\
Lucid dreams & $15 \%(14.2$ to $16.6 \%)$ & $34 \%$ \\
\hline
\end{tabular}

Table 6

Test results for latent disease.

\begin{tabular}{lllll}
\hline Virus & Positive* $^{*}$ & Positive (past) & Negative & Total Tested \\
\hline Epstein-Barr (EBV) & 40 & 309 & 231 & 580 \\
Lyme Disease & 7 & 34 & 366 & 407 \\
Cytomegalovirus (CMV) & 4 & 85 & 204 & 293 \\
\hline
\end{tabular}

* Includes both current and recent cases.

\subsubsection{Other neuropsychiatric symptoms}

The most common neuropsychiatric category was sensorimotor symptoms (prevalence of $91.44 \%, 95 \%$ confidence interval $90.48 \%$ to 92.29\%). Other neuropsychiatric symptoms included speech and language issues $(48.6 \%, 47.0 \%$ to $50.2 \%$ ), headaches $(77.0 \%, 75.4 \%$ to $78.0 \%)$, emotion and mood (88.3\%, 87.2\% to $89.3 \%)$, taste and smell (57.6\%, 56.0\% to $59.2 \%$ ), and hallucinations (23.2\%, $21.9 \%$ to $24.6 \%$ ). See Supplementary Material for detailed discussion of all sub-symptoms.

$78.6 \%$ (95\% confidence interval $84.0 \%$ to $79.9 \%$ ) of respondents experienced difficulty with sleep (Fig. 3c, Supplemental Table S19 for full prevalence data). Table 5 lists the prevalence of each sleep symptom, as well as the percentage of respondents with that symptom who also reported it as pre-existing (before COVID-19 infection).

\subsection{Special considerations}

Nearly half of respondents (43.4\%) were diagnosed with at least one condition post-acute COVID-19 infection (see Table S2 Appendix C.3).

\subsubsection{Postural orthostatic tachycardia syndrome (POTS)}

Of the 2308 patients who reported tachycardia, $72.8 \%(n=1680)$ reported being able to measure their heart rate in standing vs. sitting posture. Of those, $30.65 \%(n=515)$ reported an increase in heart rate of at least 30 BPM on standing, suggesting the possibility of Postural Orthostatic Tachycardia Syndrome (POTS, [22])

\subsubsection{Reactivation and test results for latent disease}

Since being infected with SARS-CoV-2, 2.8\% (95\% confidence interval $2.3 \%$ to $3.3 \%$ ) of respondents reported experiencing shingles (varicella zoster reactivation), 6.9\% reported current/recent Epstein-Barr virus (EBV) infection, 1.7\% reported current/recent Lyme infection, and $1.4 \%$ reported current/recent Cytomegalovirus (CMV) infection. Detailed results are shown in Table 6.

\subsubsection{Post-Exertional malaise (PEM)}

The survey asked participants whether they have experienced "worsening or relapse of symptoms after physical or mental activity during COVID-19 recovery"[23]. Borrowing from Myalgic Encephalomyelitis/Chronic Fatigue Syndrome (ME/CFS) terminology [24], this is referred to as post-exertional malaise (PEM). $89.1 \%$ of participants
(95\% confidence interval 88.0\% to 90.0\%) reported experiencing either physical or mental PEM. PEM was triggered at various time points after exertion, (Fig. 8a) and, for the majority of respondents, lasted for a few days $(68.3 \%, 66.4 \%$ to $69.6 \%$, Fig. 8 b). The distribution of severity scores (out of 10) is shown in Fig. 8c.

\subsection{Symptoms by test result}

Among respondents who received a diagnostic test (RT-PCR or antigen) for SARS-CoV-2 at any point during their illness, 1730 tested negative and 600 tested positive. The primary difference between these two groups was the time elapsed between symptom onset and testing, with a median of 6 days for those who tested positive and 43 days for those who tested negative $(p<0.001$, Mann-Whitney $\mathrm{U}$ test) (Supplemental Figure S6). Symptoms were remarkably similar between the two groups. We compared symptom prevalence among positively and negatively tested respondents, stratified by test time. Out of 203 symptoms, 203 showed no statistically significant difference ( $p>0.05$; Fisher's exact test, Bonferroni corrected). The loss of smell and taste were the only exceptions (loss of smell: 22.2\% (negative) vs $60.8 \%$ (positive), $p<0.0001 ; 21.5 \%$ loss of taste: $21.5 \%$ (negative) vs. $54.9 \%$ (positive), $p<0.0001$; Fisher's exact test, Bonferroni corrected). In addition, 683 participants tested positive for SARSCoV-2 antibodies (either IgG, IgM, or both).

Furthermore, respondents experienced similar variation in symptoms over time, despite differences in testing status. For 65 out of 66 symptoms, time courses overlapped substantially between participants with confirmed COVID-19 ( $n=1020$, positive RT-PCR, antigen, or antibody test at any point) and participants with no positive test result ( $n=2742$, Fig. 9). As above, change in smell/taste was the lone exception. Similar overlap was observed when separately comparing positively tested participants to negatively tested and untested participants (Supplemental Figures S7 and S8).

\subsection{Recovery, return to baseline}

\subsubsection{Relapses: triggers E' experience}

Patients with Long COVID can experience relapsing-remitting symptoms[7]. In this cohort, a minimum of $85.9 \%$ (84.8\% to $87.0 \%$ ) of respondents reported experiencing relapses (Fig. 10a-b). Respondents characterized their relapses as occurring in an irregular pattern (52.8\%, 95\% confidence interval $51.2 \%$ to $54.4 \%$ ) and in response to a specific trigger (52.4\%, 50.8\% to $54.0 \%$ ). The most commonly reported triggers of relapses (or of general worsening of symptoms) were physical activity $(70.7 \%, 69.2 \%$ to $72.1 \%)$, stress $(58.9 \%, 57.3 \%$ to $60.5 \%$ ), exercise (54.39\%, 52.8\% to $56.0 \%$ ), and mental activity ( $46.2 \%$, $44.7 \%$ to $47.8 \%$ ). More than a third of menstruating participants experienced relapses during (34.3\%, 32.0\% to $36.5 \%$ ) or before menstruation $(35.2 \%, 33.0 \%$ to $37.3 \%)$. 

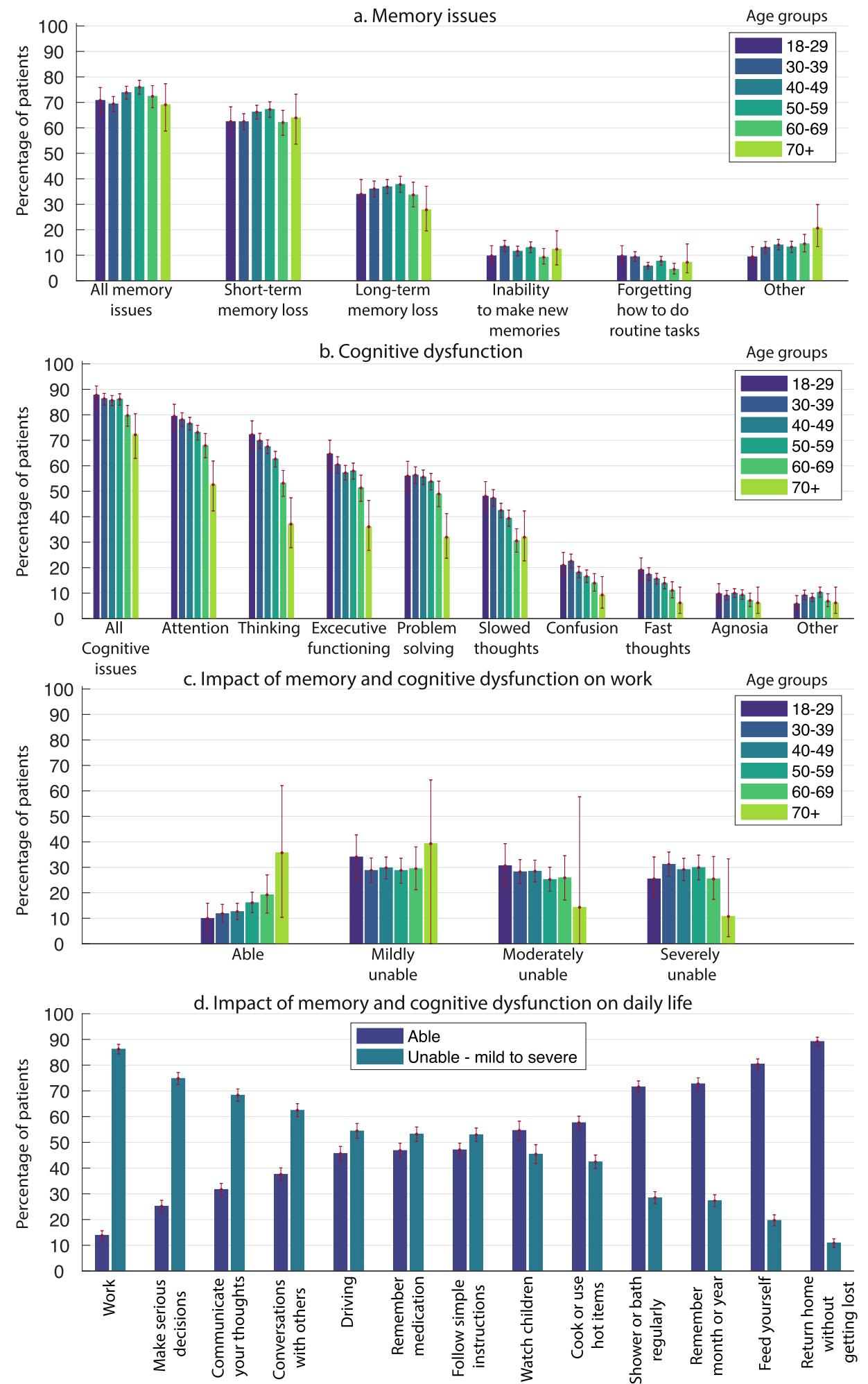

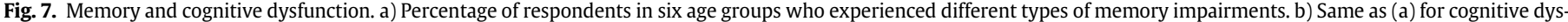

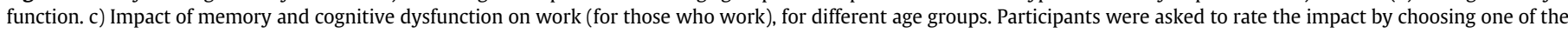

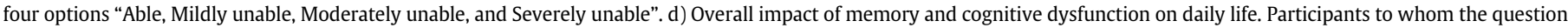
was not applicable were excluded. Error bars show bootstrap 95\% confidence intervals.

\subsubsection{Remaining symptoms after 6 months}

Only 164 out of 3762 participants (4.4\%) experienced a temporary break in symptoms (Supplemental Figure S4). The remaining participants reported symptoms continuously, until symptom resolution or up to taking the survey. A total of 2454 (65.2\%) respondents experienced symptoms for at least six months. For this population, the top remaining symptoms after six months were primarily a combination of systemic and neurological symptoms (Fig. 11a), including fatigue (80.0\%, 95\% confidence interval $78.5 \%$ to $81.6 \%$ ), post-exertional malaise $(73.3 \%, 71.5 \%$ to $75.1 \%)$, cognitive dysfunction $(58.4 \%, 56.5 \%$ to $60.2 \%$ ), sensorimotor symptoms ( $55.7 \%, 53.7 \%$ to $57.6 \%$ ), headaches (53.6\%, 51.5\% to $55.5 \%$ ), and memory issues (51.0\%, $49.1 \%$ to $53.0 \%)$. 
a. When does PEM start?

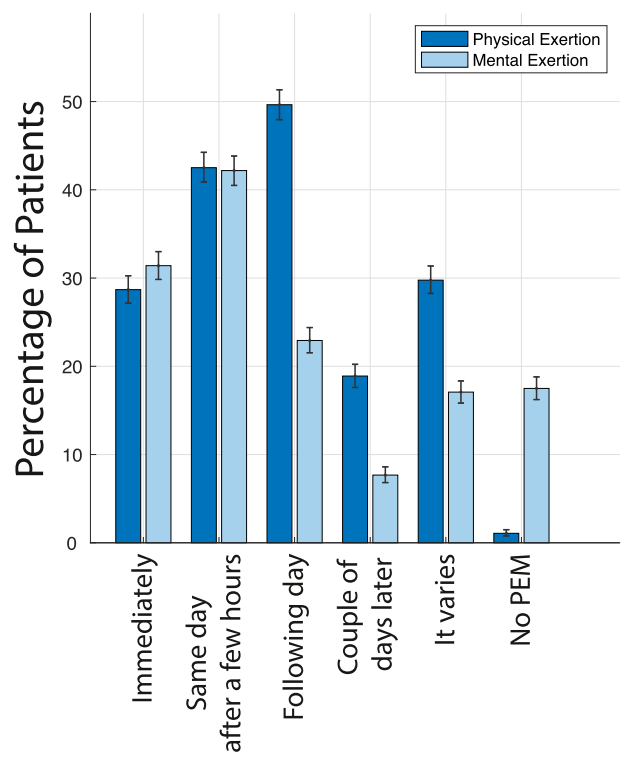

b. How long does PEM last?

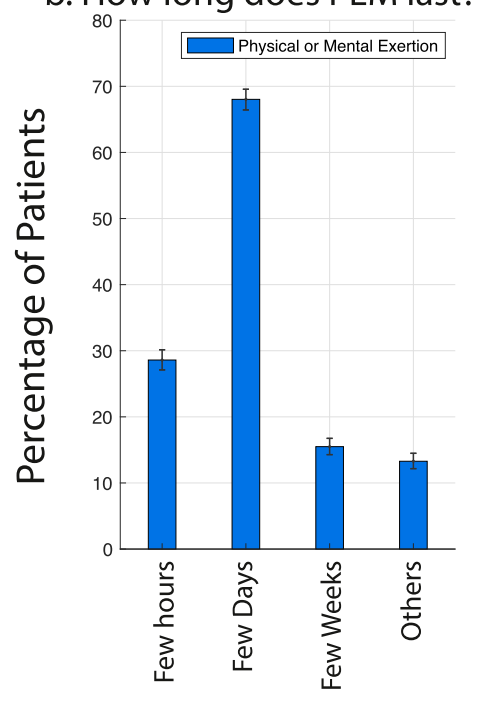

\section{c. How severe is PEM?}

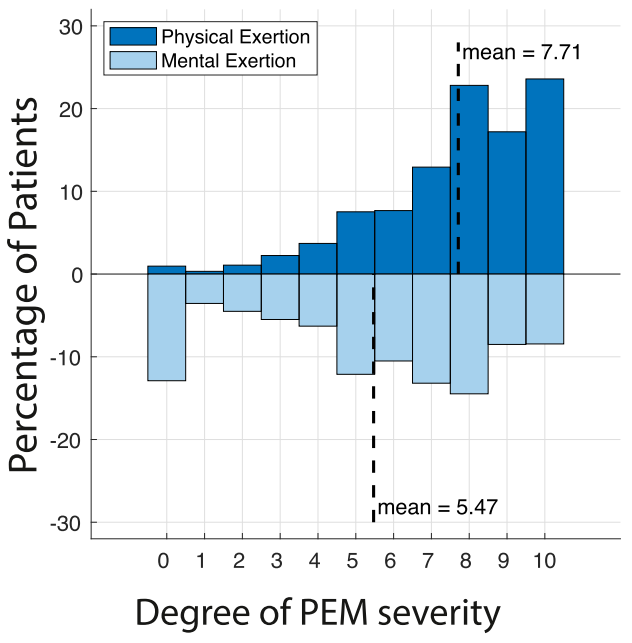

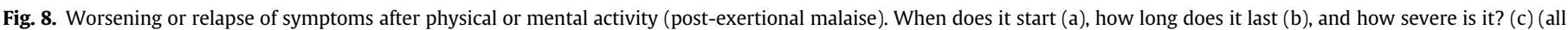
patients who experienced PEM, $n=3350$ ). Error bars are bootstrap 95\% confidence intervals.

Respondents who still experienced PEM after six months had significantly more symptoms than those who never experienced PEM, and those whose PEM resolved by month 6 (Fig. 11b, c).

\subsubsection{Fatigue assessment}

We contrasted the Fatigue Assessment Scale (FAS) scores[17,18] of unrecovered ( $n=3505$, experiencing symptoms for average of 144 days) and recovered participants ( $n=257$, experiencing symptoms for average of 91 days). On average, unrecovered participants had higher FAS scores than recovered participants (31.8 vs 22.2, $P<$ 0.001, Mann-Whitney $U$ test, Fig. 12a). 55.3\% (95\% confidence interval $49.4 \%$ to $61.5 \%$ ) of recovered participants were classified as having no fatigue. This is significantly more than the $13.2 \%$ (12.2\% to $14.4 \%, P<$ 0.001 , Mann-Whitney $U$ test, Fig. 12b) of unrecovered participants who experienced no fatigue at the time of survey. $40.7 \%$ (39.9\% to $42.3 \%$ ) of unrecovered participants were classified as experiencing extreme levels of fatigue, which was significantly higher than the $8.9 \%$ (5.8\% to $12.8 \%)$ of recovered participants in this category $(P<$ 0.001, Mann-Whitney $U$ test, Fig. 12b).

\subsubsection{Return to baseline}

Respondents were asked, "How would you rate how you feel today, on a scale of $0-100 \%$ (with $100 \%$ being your pre-COVID baseline)?" (Fig. 12c). Unrecovered participants reported a mean score of 59.2, while recovered participants reported a mean score of $86.5(p<0.001$, Mann-Whitney $U$ test). $23.1 \%$ of respondents considered "pacing" to be "significantly helpful" (out of 1788 who tried it)-a greater fraction than for other treatments reported. $18.8 \%$ found it "slightly helpful".

\subsubsection{Impact on work}

Among unrecovered respondents who worked before becoming ill, only $27.3 \%$ (95\% confidence interval $25.3 \%$ to $29.4 \%$ ) were working as many hours as they were prior to becoming ill at the time of survey, compared to $49.3 \%$ ( $40.8 \%$ to $57.9 \%$ ) of recovered respondents (see Fig. 12 d). Nearly half $(45.6 \%, 43.2 \%$ to $48.0 \%)$ of unrecovered respondents were working reduced hours at the time of the survey, and $23.3 \%$ ( $21.3 \%$ to $25.4 \%$ ) were not working at the time of the survey as a direct result of their illness. This included being on sick leave, disability leave, being fired, quitting, and being unable to find a job that would accommodate them. The remaining respondents retired, were volunteers, or did not provide enough information to determine their working status. Overall, $45.2 \%$ (42.9\% to $47.2 \%)$ of respondents reported requiring a reduced work schedule compared to pre-illness. $22.3 \%$ ( $20.5 \%$ to $24.3 \%$ ) were not working at the time of survey due to their health condition. See Appendix B for thematic analysis of participants' free text responses [25-27] on their working status (selected quotes in Appendix D).

\section{Discussion}

Results from this international online survey of 3762 individuals with suspected or confirmed COVID-19 illness suggest that Long COVID is composed of heterogeneous sequelae that often affect multiple organ systems, with impact on functioning and ability to work. To our knowledge, this represents the largest collection of symptoms identified in the Long COVID population to date. While several others have investigated Long COVID symptoms [8,28], our approach also allowed for the first representation of individual symptom trajectories over time.

Our analyses show that participants experience symptoms that are not commonly mentioned in public discussion of Long COVID $[3,29,30]$, and may benefit from further research. These include but are not limited to: anaphylaxis and new allergies, seizures, suicidality, changes in sensitivity to medication, vision loss, hearing loss, and facial paralysis. Several of these symptoms (e.g. anaphylaxis,new allergies, changes in sensitivity to medications), as well as the more commonly reported Long COVID symptoms (e.g. dizziness and tachycardia), overlap with symptoms of Mast Cell Activation Syndrome (MCAS), possibly warranting further exploration into the role of mast cells in Long COVID [31].

Dysautonomia, including Postural Orthostatic Tachycardia Syndrome (POTS), and Myalgic Encephalomyelitis/Chronic Fatigue Syndrome (ME/CFS) appear as highly possible diagnoses for this population [32]. By the time respondents took the survey, 155 had received a diagnosis of POTS, and 118 had received a diagnosis of ME/ CFS. 33.9\% of respondents who reported tachycardia measured an increase of at least 30 BPM when standing, suggesting a possible POTS diagnosis [33]. Given these findings, we suggest that all patients who present with any signs or symptoms of POTS, including tachycardia, dizziness, brain fog, or fatigue, be screened for POTS [22]. 


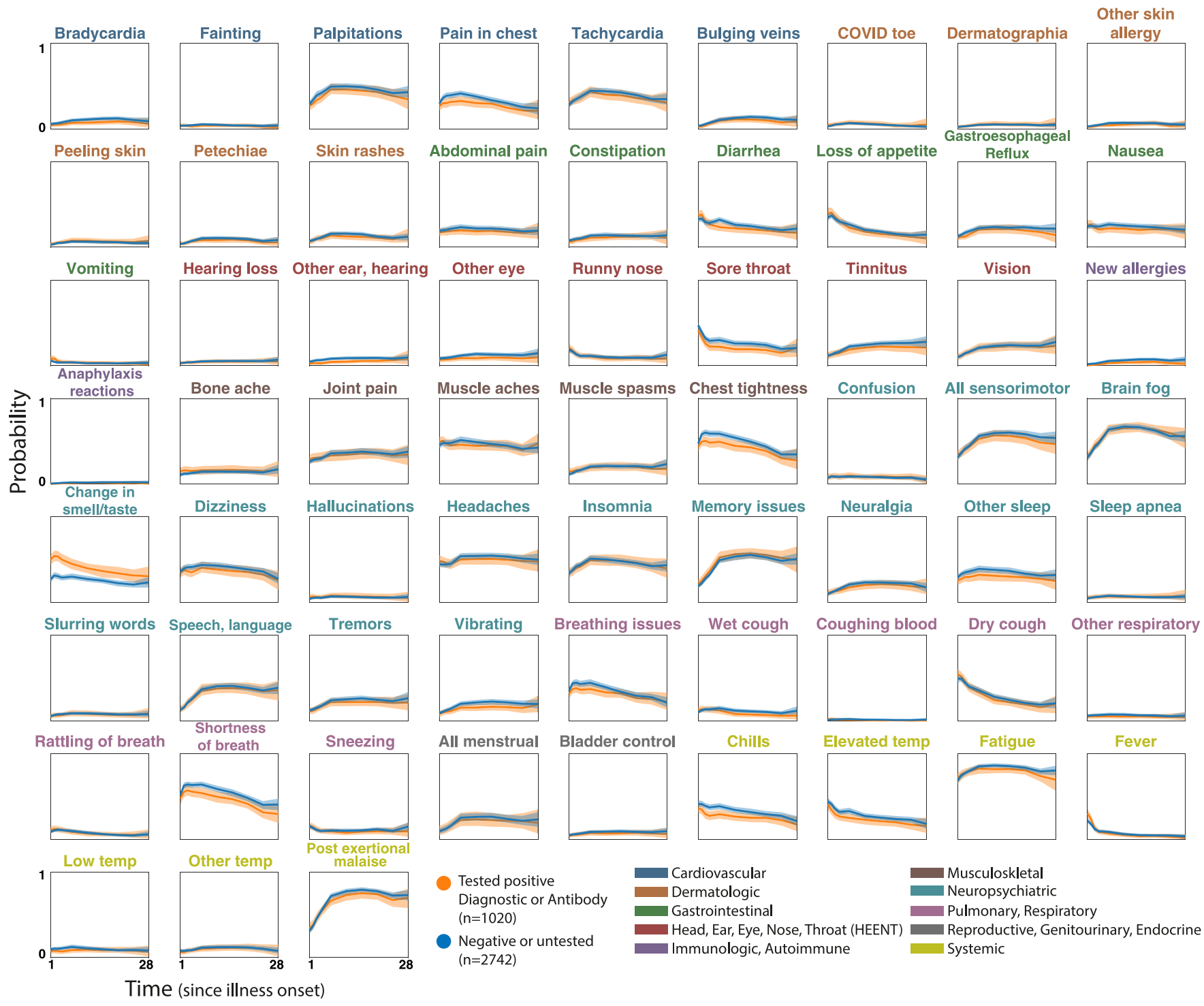

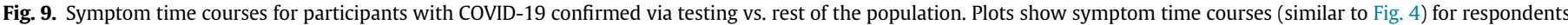

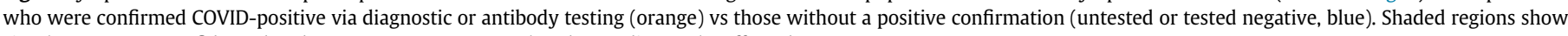
simultaneous $95 \%$ confidence bands. Symptom names are colored according to the affected organ systems.

To investigate the possible overlap with ME/CFS in this population, we asked participants to identify whether they experienced worsening of symptoms after physical or mental exertion. This is a phenomenon known as Post-Exertional Malaise (PEM), which is one of the three required symptoms for ME/CFS diagnosis along with unrefreshing sleep and a reduction in ability to engage in pre-illness levels of activity [34]. We found PEM to be highly represented in this cohort $(89.1 \%$ at any time during the course of illness, $72.2 \%$ at month 7). Intriguingly, among those still experiencing symptoms at month 6 with no PEM ( $n=707,28.8 \%$ ), fatigue was still the most common symptom.

This work highlights the wide range of neurologic symptoms experienced by patients with Long COVID. Prior studies have identified evidence of cognitive dysfunction induced by COVID-19 illness, with few studies in the non-hospitalized population [32,35]. Memory and cognitive dysfunction, experienced by over $88 \%$ of respondents, were the most pervasive and persisting neurologic symptoms in this cohort, equally common across all ages, and with substantial impact on work and daily life. Memory and cognitive dysfunction, together with other commonly reported neuropsychiatric symptoms, may point to larger neurological issues involving both the central and peripheral nervous system.

The reduced work capacity because of cognitive dysfunction, in addition to other debilitating symptoms, translated into the loss of hours, jobs, and ability to work relative to pre-illness levels. Additionally, only 55.3\% of recovered respondents had Fatigue Assessment Scores ranked as "no fatigue". This could suggest that some respondents who reported that they were no longer experiencing symptoms considered any lingering effects as part of their new health baseline. For those who returned to their job, respondents reported experiencing relapses triggered by the mental exertion and stress of work, often needing to go back on leave. This emphasizes the importance of all patients having adequate time off to recover, being able to qualify for disability benefits if long-term assistance is needed, and receiving accommodations at work including telecommuting, flexible hours, and phased returns. Lower wage earners may find it especially challenging to access accommodations and benefits, yet they are in need of protections the most to ensure financial stability [36]. Further 
a. Triggers of relapses

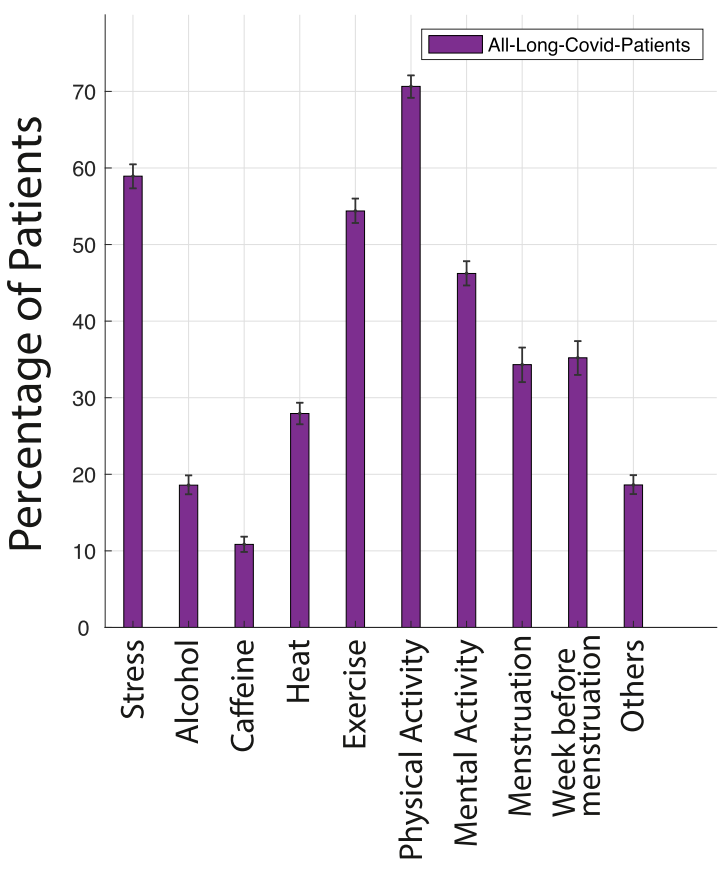

b. Experiences with relapses and symptom course

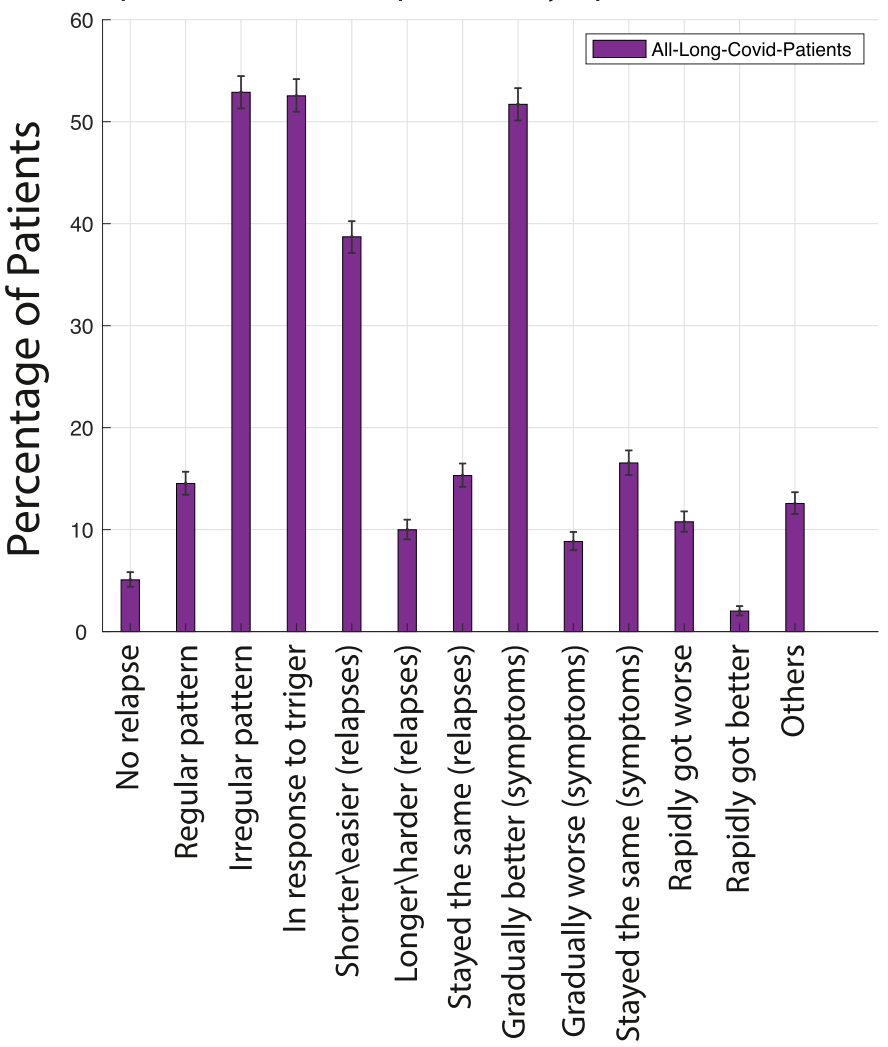

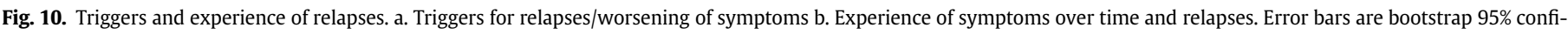
dence intervals.

investigation could be done to measure the quality of life after Long COVID across socioeconomic strata.

Overall, these findings suggest that the morbidity of COVID-19 illness has been greatly overlooked. Patients experience multisystem symptoms for over seven months, resulting in significant impact to their lives and livelihoods.

Our analysis confirms prior findings that, with the exception of change to smell and taste, symptoms are not significantly different between those who test positive for SARS-CoV-2 and those who test negative (or have not been tested), but who otherwise show strongly suggestive symptoms [7,37]. The sensitivity of diagnostic tests may depend on the primer/probe sets [38,39]. Furthermore, the likelihood of false negatives increases after day 3 of symptom onset, when the false negative rate is $20 \%$, reaching $66 \%$ by day 21 [40]. This reinforces the need for early testing in patients with suspected SARS-CoV-2 infection, given that up to $54 \%$ of patients could have an initial RTPCR false-negative result [41]. The importance of early testing was reflected in this cohort as well: the median number of days between first experiencing symptoms and being tested was 6 days for those who tested positive and 43 days for those who tested negative. Access to adequate diagnostic tests in the early stages of the pandemic was notably limited, which likely contributed to respondents in this cohort being unable to be tested and/or being tested later in their illness [42]. The site of sample collection, e.g. nasopharyngeal swab sampling vs. sputum testing [43], or stool vs. respiratory specimens [44] can also play an important role in testing accuracy [43]. Regarding antibody testing, it has been reported that antibody levels decrease with time [44,45], that males are likely to retain antibodies longer than females [46], and that antibody tests can be less accurate for females [47]. These results may be relevant to our cohort, of whom the majority was female. There is also evidence that patients with neurological symptoms but minimal respiratory symptoms may fail to seroconvert [33]. Together, these findings indicate that absent or negative SARS-CoV-2 diagnostic and antibody tests should not be used as an indicator to rule out Long COVID in patients who otherwise have suggestive symptoms [37,48-50]. Further investigation is needed to understand why some Long COVID patients test positive and others do not, despite having similar symptom courses.

While the majority of participants did not report receiving a positive SARS-CoV-2 diagnostic or antibody test result, our analysis of symptoms in confirmed and suspected COVID-19 groups indicates that this is only a limitation in the sense that diagnostic serology is lacking. Removing suspected COVID-19 participants from our analysis does not change the results.

The retrospective nature of the study exposes the possibility of recall bias, which could impact the reliability of symptom prevalence estimates. Because participants were asked to report any symptoms experienced within the designated time periods, both overreporting and underreporting of symptoms are possible. As the survey was distributed in online support groups, there exists a sampling bias toward Long COVID patients who joined support groups and were active participants of the groups at the time the survey was published. The effort to complete the survey may have deterred some respondents who experienced cognitive dysfunction, or were no longer ill and did not have incentives to participate. Furthermore, most respondents (91.6\%) had not been hospitalized. The severity of illness that the survey captured may not be representative of the average Long COVID patient because of these issues. Additionally, despite eight translations and inclusive outreach efforts, the demographics were strongly skewed towards English speaking (91.9\%), white (85.3\%), and higher socioeconomic status (see Figure S1). Moreover, the study required respondents to have stable internet and email addresses, which may have excluded participants who lacked access and/or had low digital literacy. In future studies, more outreach and partnerships with 
a. Remaining symptoms after month 6 (prevalence $>30 \%$ )

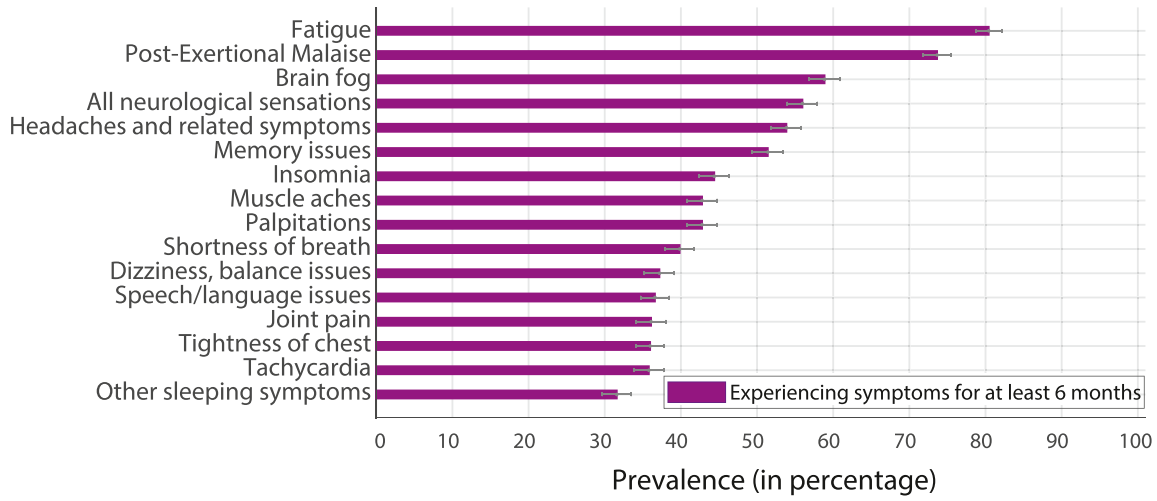

b. Remaining symptoms after month 6, for PEM vs No PEM groups (prevalence $>30 \%$ )

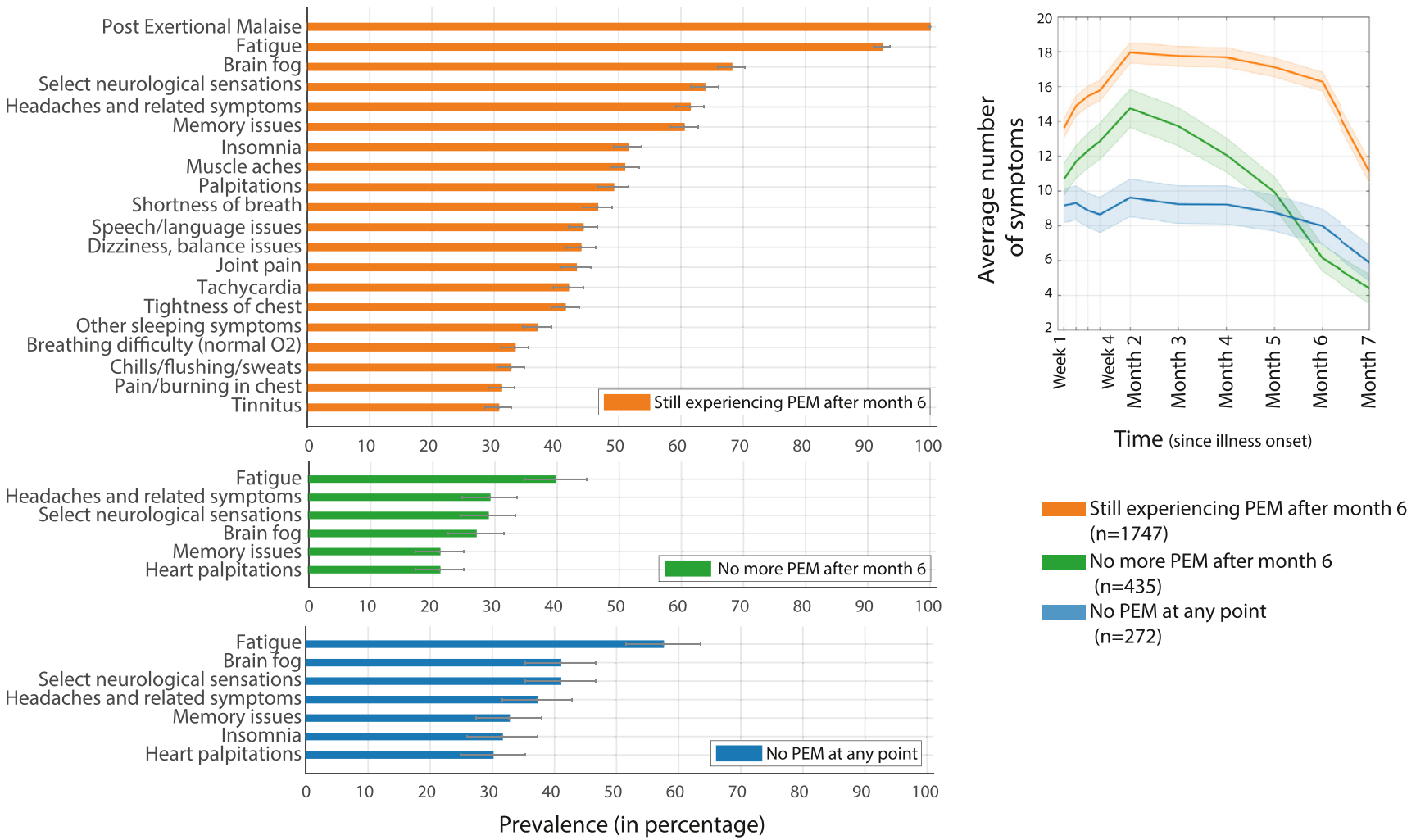

Fig. 11. Remaining symptoms after six months. a) Symptoms remaining after six months. b) Symptoms remaining after six months for respondents still experiencing PEM after six months (orange), respondents not experiencing PEM after six months (green), and respondents who never experienced PEM (blue). c) Average number of symptoms over time for each group in (b). Error bars are bootstrap 95\% confidence intervals.

diverse groups, low-income communities, and communities of color can be established to counter sampling bias.

As a result of the above limitations, the study may not be representative of the entire Long COVID population or their experiences.

We suggest that the results laid forth be considered only in the context of this study; extrapolation of the results to all patients with Long COVID requires caution.

\section{Declaration of Competing Interest}

All authors have completed the ICMJE uniform disclosure form and declare: no support from any organization for the submitted work. All authors except HED and GSA declare no financial relationships with any organization that might have an interest in the submitted work in the previous three years, no other relationships or activities that could appear to have influenced the submitted work. HED reports personal fees (\$500 speaking fee) from Council for
Medical Specialty Society, outside the submitted work. GSA reports personal fees ( $\$ 1000$ speaking fee) from Council for Medical Specialty Society and Karolinska Institute, outside the submitted work.

\section{Acknowledgments}

We would like to thank the admin team at Body Politic COVID-19 Slack Support Group. We owe our success to their support in distributing the survey, connecting us with each other and to volunteers. We would like to thank all respondents for their efforts in contributing to the study and providing feedback. In particular, we would like to thank Rachel Robles for her assistance in data cleaning, Monique Jackson for illustrations, Jared Mercier for IT support. We would like to thank our translators: Oksana Zinchenko (Russian); Emeline Chavernac (French); Maarten Steenhagen and Red Team C19 NL Community (Dutch); Luisa Pereira, Lucía Landa, Maria Teresa Cabañero, Daniel Hernandez Diaz, Brenda Valderrama, and Lorena Ramírez- 
a. Fatigue Assessment Scale (FAS)

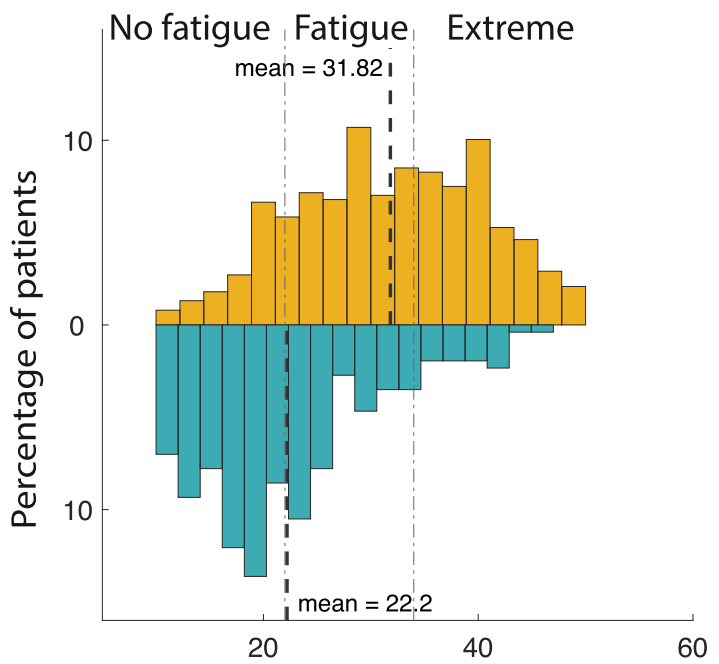

Fatigue Assessment Score

b. FAS categories (No fatigue, Fatigue \& Extreme)

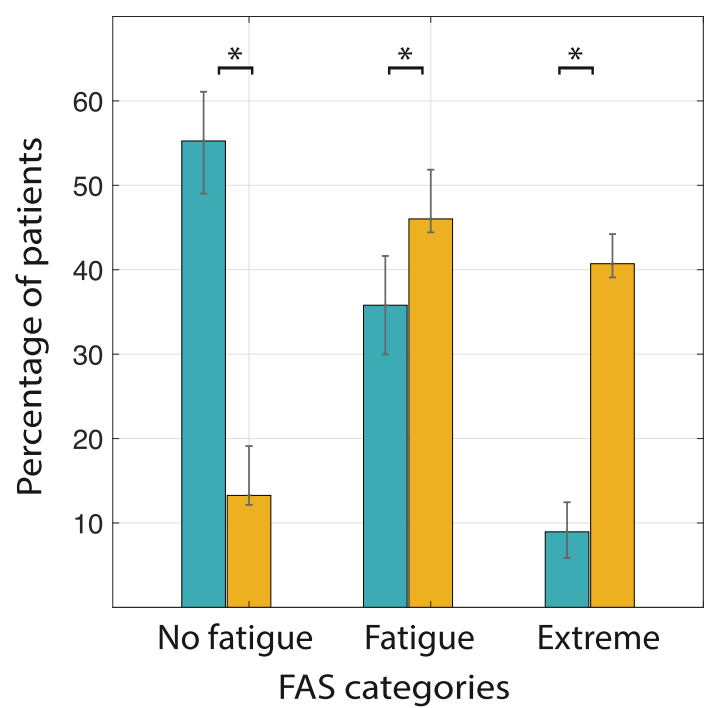

\section{Not recovered} Recovered

\section{c. Return to pre-COVID baseline}

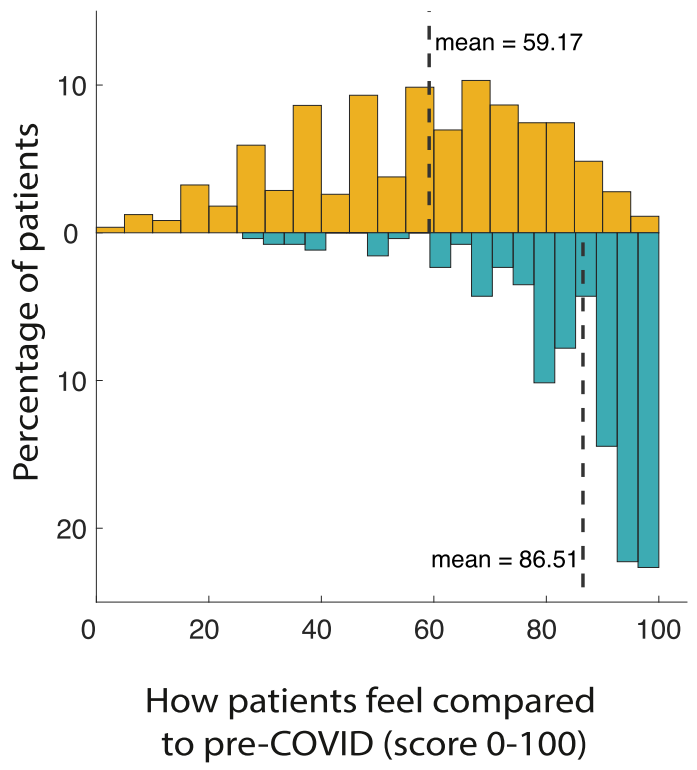

\section{d. LONG COVID impact on work}

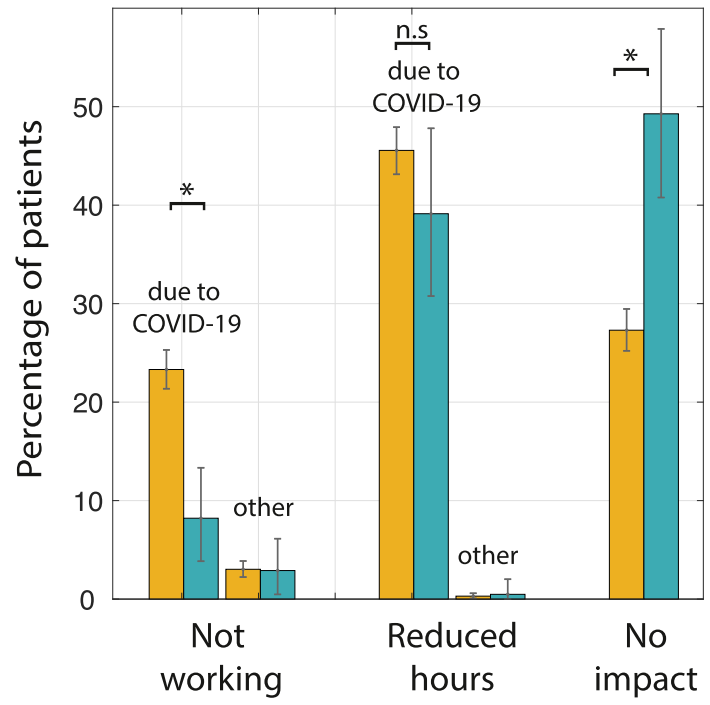

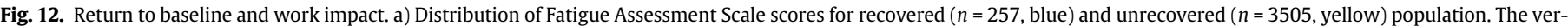

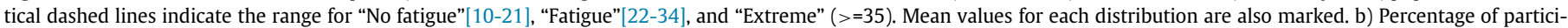

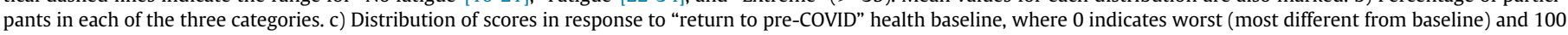
indicates best (most similar to baseline). d) Working status due to COVID-19. Error bars show 95\% simultaneous confidence interval.

Nícoles (Spanish); Liliana Vagnoni (Italian); Victor Pedrosa, Monica Malta, and Noris Kern (Portugues); Juno Simorangkir (Indonesian); and Rawan Alsubaie, Sarah Mitkees, Mohamed Abdelhack, Dalia Aroury, Luna Aroury, and Ihsan Kaadan (Arabic). We would also like to acknowledge Dr. Alka Gupta of WCMC for her guidance and involvement in the ethics approval process. Finally, we thank the Long COVID community and allies in the ME/CFS community for their knowledge and support in patient-driven research.

\section{Author contributions}

AA, GSA, HED, LM, YR, and HW conceived the project and designed the survey. HED accessed and cleaned the raw data. AA, GSA, HED, RJL, and LM analyzed the quantitative data. AA and RJL performed the statistical analyses. HED, LM, and HW analyzed the qualitative data. AA and RJL created the figures. HW and AA created the tables. JPA and YR provided medical input. AA, GSA, HED, RJL, LM, SR, YR, and HW wrote the manuscript, with extensive comments from JPA. The corresponding author attests that all listed authors meet authorship criteria and that no others meeting the criteria have been omitted. AA, GSA, HED, RJL, LM, YR, and HW contributed equally to this work. AA is the Principal Investigator and guarantor.

The corresponding author (AA) affirms that the manuscript is an honest, accurate, and transparent account of the study being reported; that no important aspects of the study have been omitted; and that any discrepancies from the study as planned have been explained. 


\section{Funding}

All authors contributed voluntarily to this work. The cost of survey hosting (on Qualtrics) and publication fee was covered by AA's research grant (Wellcome Trust/Gatsby Charity via Sainsbury Wellcome center, UCL).

\section{Data sharing statement}

The data collected for this study, including anonymized individual patient data and a data dictionary defining each field in the data set will be made publicly available. Interested parties can contact the corresponding author (AA).

\section{Supplementary materials}

Supplementary material associated with this article can be found, in the online version, at doi:10.1016/j.eclinm.2021.101019.

\section{References}

[1] Alwan NA. Surveillance is underestimating the burden of the COVID-19 pandemic. The Lancet 2020 Sep;396(10252):e24

[2] The prevalence of long COVID symptoms and COVID-19 complications [Internet]. Office for national statistics. [cited 2020 Dec 17]. Available from: https://www. ons.gov.uk/news/statementsandletters/ theprevalenceoflongcovidsymptomsandcovid19complications.

[3] Petersen MS, Kristiansen MF, Hanusson KD, Danielsen ME, áSteig B, Gaini S, et al. Long COVID in the Faroe Islands - a longitudinal study among non-hospitalized patients. Clinical Infect Dis [Internet] 2020 Nov 30 [cited 2020 Dec 17]; (ciaa1792). Available from:. doi: 10.1093/cid/ciaa1792.

[4] Arnold DT, Hamilton FW, Milne A, Morley A, Viner J, Attwood M, et al. Patient outcomes after hospitalisation with COVID-19 and implications for follow-up; results from a prospective UK cohort. medRxiv 2020 Aug 14;2020.08.12.20173526.

[5] Carfì A, Bernabei R, Landi F. Gemelli Against COVID-19 post-acute care study group. Persistent symptoms in patients after acute COVID-19. JAMA 2020;324 (6):603-5 Aug 11.

[6] Mitrani RD, Dabas N, Goldberger JJ. COVID-19 cardiac injury: implications for long-term surveillance and outcomes in survivors. Heart Rhythm 2020;17 (11):1984-90 Nov 1

[7] Assaf G, Davis H, McCorkell L, Wei H, Brooke O, Akrami A, et al. What does COVID19 recovery actually look like? An analysis of the prolonged COVID-19 symptoms survey by patient-led research team [Internet]; Available from: https://patientresearchcovid19.com/research/report-1/.

[8] Michelen M, Manoharan L, Elkheir N, Cheng V, Dagens D, Hastie C, et al. Characterising long-term covid-19: a rapid living systematic review. medRxiv. Dec 9;2020.12.08.20246025

[9] Al-Aly Z, Xie Y, Bowe B. High-dimensional characterization of post-acute sequalae of COVID-19. Nature 2021:1-8 Apr 22.

[10] Callard F, Perego E. How and why patients made Long Covid. Soc Sci Med 2020:113426 Oct 7 .

[11] Tenforde MW, Kim SS, Lindsell CJ, Rose EB, Shapiro NI, Files DC, et al. Symptom duration and risk factors for delayed return to usual health among outpatients with COVID-19 in a multistate health care systems network - United States, March-June 2020. MMWR Morbid. Mortal. Weekly Report 2020;69(30):993-8 Jul.

[12] Overview | COVID-19 rapid guideline: managing the long-term effects of COVID19 | guidance | nice [Internet]. NICE; 2020. [citedDec 19]. Available from: https:/| www.nice.org.uk/guidance/NG188.

[13] CDC. Healthcare Workers [Internet]. Centers for disease control and prevention. [cited 2021 May 17]. Available from: https://www.cdc.gov/coronavirus/2019ncov/hcp/clinical-care/post-covid-conditions.html.

[14] McCorkell L, S. Assaf G, E. Davis H, Wei H, Akrami A. Patient-Led Research Collaborative: embedding patients in the Long COVID narrative. PAIN Reports 2021;6(1): e913.

[15] Greenhalgh T, Knight M, A'Court C, Buxton M, Husain L. Management of postacute covid-19 in primary care. BMJ 2020 Aug;370:m3026.

[16] Ladds E, Rushforth A, Wieringa S, Taylor S, Rayner C, Husain L, et al. Persistent symptoms after Covid-19: qualitative study of 114 "long Covid" patients and draft quality principles for services. BMC Health Serv Res 2020 Dec;20(1):1144.

[17] Shahid A, Wilkinson K, Marcu S, Shapiro CM. Fatigue assessment scale (FAS) In: Shahid A, Wilkinson K, Marcu S, Shapiro CM, editors. STOP, that and one hundred other sleep scales [Internet]. New York, NY: Springer New York; 2011. [cited
2020 Dec 19]. p. 161-2. Available from: http://link.springer.com/10.1007/978-14419-9893-4 33 .

[18] Vries J de, Michielsen H. Heck GLV, Drent M. Measuring fatigue in sarcoidosis: the fatigue assessment scale (FAS). Br J Health Psychol 2004;9(3):279-91.

[19] Kleinbaum DG, Klein M. Survival analysis: a self-learning text, third edition [Internet]. 3rd Ed. New York: Springer-Verlag; 2012. [cited 2020 Dec 16]. (Statistics for Biology and Health). Available from: https://www.springer.com/gp/book/ 9781441966452.

[20] Dhillon I.S., Modha D.S. Concept decompositions for large sparse text data using clustering.:31.

[21] Efron B. Better bootstrap confidence intervals. J Am Stat Assoc 1987;82(397):171 85

[22] Agarwal AK, Garg R, Ritch A, Sarkar P. Postural orthostatic tachycardia syndrome. Postgrad Med J 2007 Jul;83(981):478-80.

[23] Salmon-Ceron D, Slama D, Broucker TD, Karmochkine M, Pavie J, Sorbets E, et al Clinical, virological and imaging profile in patients with prolonged forms of COVID-19: a cross-sectional study. J Infection [Internet] 2020 Dec 4 [cited 2020 Dec 17];0(0). Available from: https://www.journalofinfection.com/article/S01634453(20)30762-3/abstract.

[24] Stussman B, Williams A, Snow J, Gavin A, Scott R, Nath A, et al. Characterization of post-exertional malaise in patients with myalgic encephalomyelitis/chronic fatigue syndrome. Front Neurol [Internet] 2020 Sep 18 [cited 2020 Dec 17];11. Available from: https://www.ncbi.nlm.nih.gov/pmc/articles/PMC7530890/.

[25] Corden A, Sainsbury R. University of York, social policy research unit. Using verbatim quotations in reporting qualitative social research: researchers' views. York: University of York; 2006.

[26] Bird S, Loper E, Klein E. Natural language processing with Python. O’Reilly Media Inc; 2009.

[27] Braun V, Clarke V. Using thematic analysis in psychology. Qualitative research in psychology [Internet]. [cited 2020 Dec 23];3(2). Available from: https://uwerepository.worktribe.com/output/1043060.

[28] Sudre CH, Murray B, Varsavsky T, Graham MS, Penfold RS, Bowyer RC, et al Attributes and predictors of long-COVID: analysis of COVID cases and their symptoms collected by the COVID symptoms study app. medRxiv. Oct 21:2020.10.19.20214494.

[29] CDC. Coronavirus Disease. (COVID-19) - symptoms [Internet]. centers for disease control and prevention. 2020 [cited 2020 Dec 24]. Available from: https://www. cdc.gov/coronavirus/2019-ncov/symptoms-testing/symptoms.html.

[30] Symptoms of coronavirus (COVID-19) [Internet]. nhs.uk. 2020 [cited 2020 Dec 24]. Available from: https://www.nhs.uk/conditions/coronavirus-covid-19/symptoms/

[31] Afrin LB, Weinstock LB, Molderings GJ. Covid-19 hyperinflammation and postCovid-19 illness may be rooted in mast cell activation syndrome. Int J Infect Dis 2020;100:327-32 Nov.

[32] NIH workshop on post-acute sequelae of COVID-19 (Day 2) [Internet]. Available from: https://videocast.nih.gov/watch=38879

[33] Anjum I, Sohail W, Hatipoglu B, Wilson R. Postural orthostatic tachycardia syndrome and its unusual presenting complaints in women: a literature minireview. Cureus [Internet]; 2020. [citedDec 18];10(4). Available from: https://www.ncbi. nlm.nih.gov/pmc/articles/PMC5988200/.

[34] IOM 2015 Diagnostic Criteria | Diagnosis | Healthcare Providers | Myalgic Encephalomyelitis/Chronic Fatigue Syndrome (ME/CFS) | CDC [Internet]. 2019 [cited 2020 Dec 18]. Available from: https://www.cdc.gov/me-cfs/healthcare-providers/ diagnosis/iom-2015-diagnostic-criteria.html

[35] Ding H, Yin S, Cheng Y, Cai Y, Huang W, Deng W. Neurologic manifestations of nonhospitalized patients with COVID-19 in Wuhan, China. Med Comm 2020;1 (2):253-6.

[36] Shuey KM, Jovic E. Disability accommodation in nonstandard and precarious employment arrangements. Work Occup 2013 May;40(2):174-205.

[37] Tabacof L, Tosto-Mancuso J, Wood J, Cortes M, Kontorovich A, McCarthy D, et al, Post-acute COVID-19 syndrome negatively impacts health and wellbeing despite less severe acute infection. medRxiv. Nov 6;2020.11.04.20226126.

[38] Liu X, Feng J, Zhang Q, Guo D, Zhang L, Suo T, et al. Analytical comparisons of SARS-COV-2 detection by qRT-PCR and ddPCR with multiple primer/probe sets. Emerg Microbes Infect 2020 Jan;9(1):1175-9.

[39] Watson J, Whiting PF, Brush JE. Interpreting a covid-19 test result. BM] 2020 May;369:m1808

[40] Kucirka LM, Lauer SA, Laeyendecker O, Boon D, Lessler J. Variation in false-negative rate of reverse transcriptase polymerase chain reaction-based SARS-CoV-2 tests by time since exposure. Ann Intern Med [Internet] 2020 May 13 [cited 2020 Dec 17] Available from https://www.ncbi.nlm.nih.gov/pmc/articles/PMC7240870/.

[41] Arevalo-Rodriguez I, Buitrago-Garcia D, Simancas-Racines D, Zambrano-Achig P, Campo R del, Ciapponi A, et al. False-negative results of initial RT-PCR assays for COVID-19: a systematic Review. medRxiv. 2020.04.16.20066787.

[42] Sharfstein JM, Becker SJ, Mello MM. Diagnostic testing for the novel coronavirus JAMA 2020 Apr;323(15):1437.

[43] Tang A, Tong Z-D, Wang H-L, Dai Y-X, Li K-F, Liu J-N, et al. Detection of novel coronavirus by RT-PCR in stool specimen from asymptomatic child, china. emerg infect dis, 26; 2020. Junp. 1337-9.

[44] Kuehn BM. Health workers' antibody levels wane after SARS-CoV-2 infection. JAMA 2021;325(2):122. Jan 12. 
[45] Gaebler C, Wang Z, Lorenzi JCC, Muecksch F, Finkin S, Tokuyama M, et al. Evolution of antibody immunity to SARS-CoV-2. Nature 2021 Jan:1-10.

[46] Williams J, Watkeys L, Nash J, Whelan C, Davies A, Evans J, et al. A two-phase, single cohort study of COVID-19. Antibody Sera-Surveillance 2021:6.

[47] Vashisht R, Patel A, Crews BO, Garner OB, Dahm L, Wilson C, et al. Age- and sexassociated variations in the sensitivity of serological tests among individuals infected with SARS-CoV-2. JAMA Netw Open 2021 Feb;4(2):e210337.

[48] Hampshire A, Trender W, Chamberlain SR, Jolly A, Grant JE, Patrick F, et al. Cognitive deficits in people who have recovered from COVID-19 relative to controls: an $\mathrm{N}=84,285$ online study. medRxiv. 2020.10.20.20215863.
[49] Goërtz YMJ, Herck MV, Delbressine JM, Vaes AW, Meys R, Machado FVC, et al. Persistent symptoms 3 months after a SARS-CoV-2 infection: the post-COVID-19 syndrome? ERJ Open Research [Internet] 2020 Jan 1 [cited 2020 Dec 19]; Available from: https://openres.ersjournals.com/content/early/2020/09/01/ 23120541.00542-2020.

[50] Vaes AW, Machado FVC, Meys R, Delbressine JM, Goertz YMJ, Van Herck M, et al. Care dependency in non-hospitalized patients with COVID-19. J Clin Med 2020;9 (9):2946. Sep. 